\title{
In situ sprayed bioresponsive immunotherapeutic gel for post-surgical cancer treatment
}

\author{
Qian Chen ${ }^{1,2,3}$, Chao Wang ${ }^{3}$, Xudong Zhang ${ }^{1,2}$, Guojun Chen ${ }^{1,2}$, Quanyin $\mathrm{Hu}^{1,2,3}$, Hongjun $\mathrm{Li}^{4}$, \\ Jinqiang Wang ${ }^{3}$, Di Wen ${ }^{1,2}$, Yuqi Zhang ${ }^{3}$, Yifei Lu ${ }^{3,5}$, Guang Yang ${ }^{1,2}$, Chen Jiang ${ }^{5}$, Jun Wang ${ }^{4}$, \\ Gianpietro Dotti ${ }^{6}$ and Zhen $\mathrm{Gu} \oplus^{1,2,3,7,8 \star}$
}

\begin{abstract}
Cancer recurrence after surgical resection remains a significant cause of treatment failure. Here, we have developed an in situ formed immunotherapeutic bioresponsive gel that controls both local tumour recurrence after surgery and development of distant tumours. Briefly, calcium carbonate nanoparticles pre-loaded with the anti-CD47 antibody are encapsulated in the fibrin gel and scavenge $\mathrm{H}^{+}$in the surgical wound, allowing polarization of tumour-associated macrophages to the M1-like phenotype. The released anti-CD47 antibody blocks the 'don't eat me' signal in cancer cells, thereby increasing phagocytosis of cancer cells by macrophages. Macrophages can promote effective antigen presentation and initiate $T$ cell mediated immune responses that control tumour growth. Our findings indicate that the immunotherapeutic fibrin gel 'awakens' the host innate and adaptive immune systems to inhibit both local tumour recurrence post surgery and potential metastatic spread.
\end{abstract}

$\mathrm{D}$ espite improvements in surgical techniques, local residual tumour micro-infiltration and circulating tumour cells continue to cause tumour recurrence after resection ${ }^{1-3}$. Furthermore, perioperative trauma-associated inflammation can promote tumour recurrence by accelerating local growth or tumour spread $^{4-7}$. Chemotherapy and radiotherapy are often used after surgery to prevent both local and metastatic tumour recurrence, but these therapies often cause toxicities ${ }^{8}$. More recently, immunotherapy has been considered to inhibit cancer recurrence and metastasis effectively ${ }^{9-13}$.

Macrophages are cellular components of the innate immunity that phagocytize foreign substances and present them to $\mathrm{T}$ lymphocytes (T cells) without requiring specific 'self-signal' proteins expressed on the cell surface ${ }^{14-16}$. However, cancer cells can escape macrophage recognition via upregulation of the integrin-associated protein (IAP) - CD47-which provides the antiphagocytic 'don't eat me signal ${ }^{17-19}$. Blocking the interaction of $\mathrm{CD} 47$ with its ligand, signal regulatory protein- $\alpha$ (SIRP $\alpha$ ), which is expressed on macrophages, dendritic cells and neutrophils ${ }^{20}$, activates phagocytic cells and promotes their capacity to phagocytize cancer cells. At least three CD47 antagonists are currently being tested in clinical studies, but the occurrence of anaemia and thrombocytopenia caused by systemic administration of these antagonists remains a concern ${ }^{21-24}$. Efforts to avoid these severe side effects are essential to make CD47blocking immunotherapies clinically applicable.

Tumour-associated macrophages (TAMs) account for a substantial fraction of tumour-infiltrating immune cells ${ }^{25,26}$. TAMs differentiate into separate functional phenotypes, denoted classically activated macrophages (the M1-like phenotype) and alternatively activated macrophages (the M2-like phenotype) ${ }^{27}$. The M1-like
TAMs overexpress major histocompatibility complex class I and class II molecules, which play critical roles in antigen presentation. Conversely, M2-like TAMs exert pro-tumorigenic activities ${ }^{28,29}$ and respond to different tumour determining factors, in particular low $\mathrm{pH}$ values $^{30}$. Infiltration of M2-like TAMs frequently correlates with tumour invasion, metastases, angiogenesis, $\mathrm{T}$ cell suppression and poor clinical outcome $\mathrm{e}^{31-33}$. Thus, targeting M2-like TAMs could be critical to alter the tumour microenvironment (TME) into a more permissive environment for the function of immune cells.

In this Article we report a sprayed bioresponsive immunotherapeutic fibrin gel that has been engineered to inhibit local tumour recurrence after surgery and development of distant tumours. The fibrin gel is a US Food and Drug Administration approved material, and is formed by the interaction of fibrinogen and thrombin. We leverage the unique merits of fibrin gel here, including excellent biocompatibility, a convenient sprayable administration method for post-surgical treatment, and the ability to promote wound healing by creating a temporary shield to connect and protect injured tissue $^{34}$. Biocompatible $\mathrm{CaCO}_{3}$ nanoparticles are incorporated into the fibrin gel to serve as a release reservoir of immunomodulatory therapeutics as well as a proton scavenger to modulate the acidity of the tumour environment ${ }^{35,36}$. The fibrinogen solution containing anti$\mathrm{CD} 47$ antibody-loaded $\mathrm{CaCO}_{3}$ nanoparticles $\left(\mathrm{aCD} 47 @ \mathrm{CaCO}_{3}\right)$ and thrombin solution can be quickly sprayed and mixed within the tumour resection cavity after surgery to form an immunotherapeutic fibrin gel in situ (Fig. 1a). We demonstrate that $\mathrm{CaCO}_{3}$ nanoparticles can gradually dissolve and release the encapsulated aCD47 in the acidic and inflamed TME, thus promoting the activation of M1-type TAMs, inducing macrophage phagocytosis of cancer cells via blockade of the CD47 and SIRP $\alpha$ interaction as well as boosting

'Department of Bioengineering, University of California, Los Angeles, CA, USA. ${ }^{2}$ California NanoSystems Institute, University of California, Los Angeles, CA, USA. ${ }^{3}$ Joint Department of Biomedical Engineering, University of North Carolina at Chapel Hill and North Carolina State University, Raleigh, NC, USA. ${ }^{4}$ National Engineering Research Center for Tissue Restoration and Reconstruction, and School of Biomedical Science and Engineering, South

China University of Technology, Guangzhou, Guangdong, China. ${ }^{5}$ Key Laboratory of Smart Drug Delivery, Ministry of Education, State Key Laboratory of Medical Neurobiology, Department of Pharmaceutics, School of Pharmacy, Fudan University, Shanghai, China. 'Lineberger Comprehensive Cancer Center, University of North Carolina, Chapel Hill, NC, USA. ${ }^{7}$ Jonsson Comprehensive Cancer Center, University of California, Los Angeles, CA, USA.

${ }^{8}$ Center for Minimally Invasive Therapeutics, University of California, Los Angeles, CA, USA. *e-mail: guzhen@ucla.edu 
a

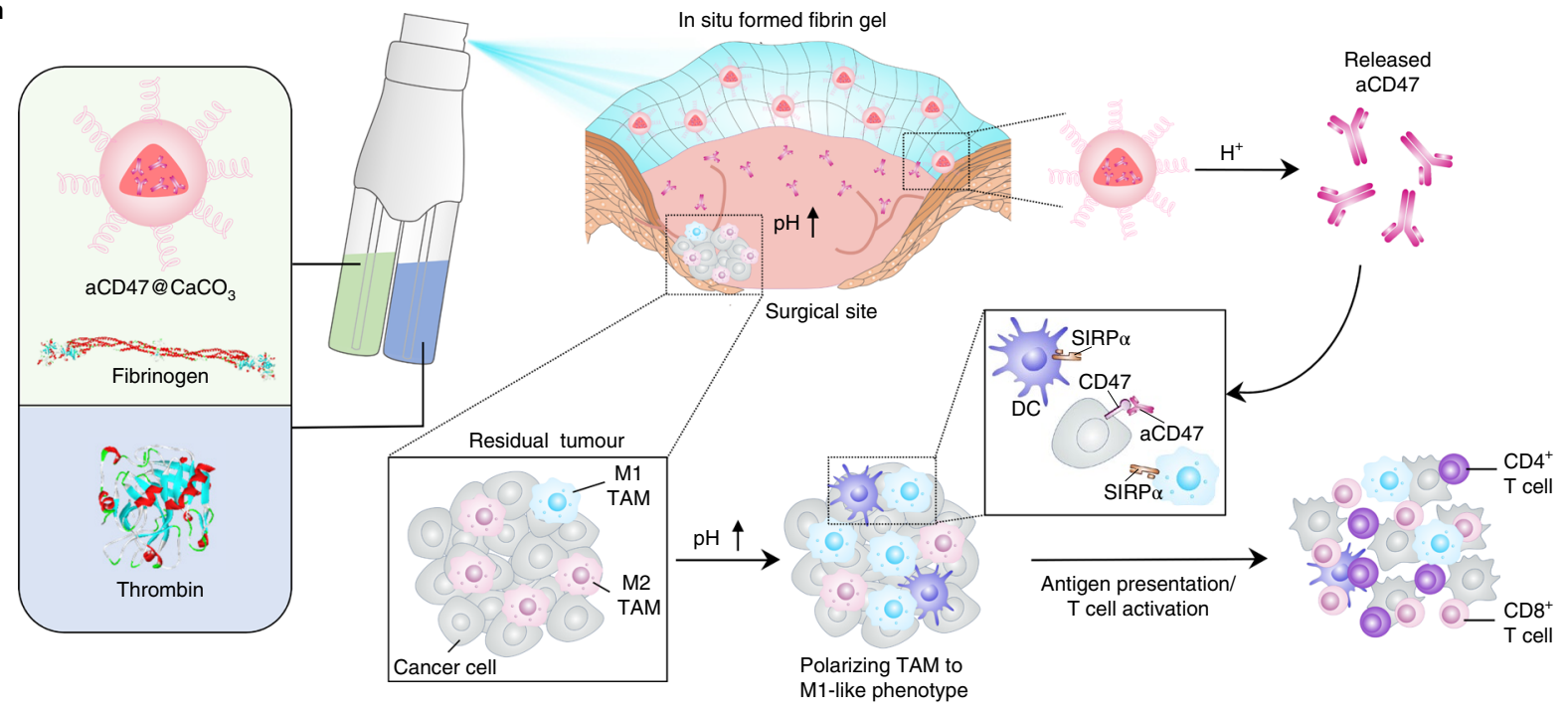

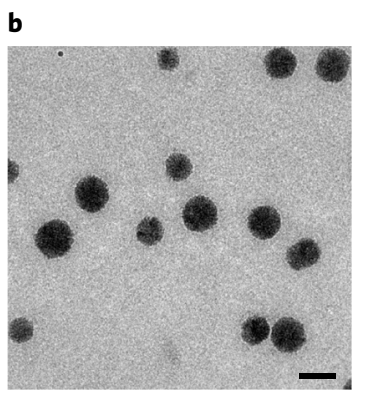
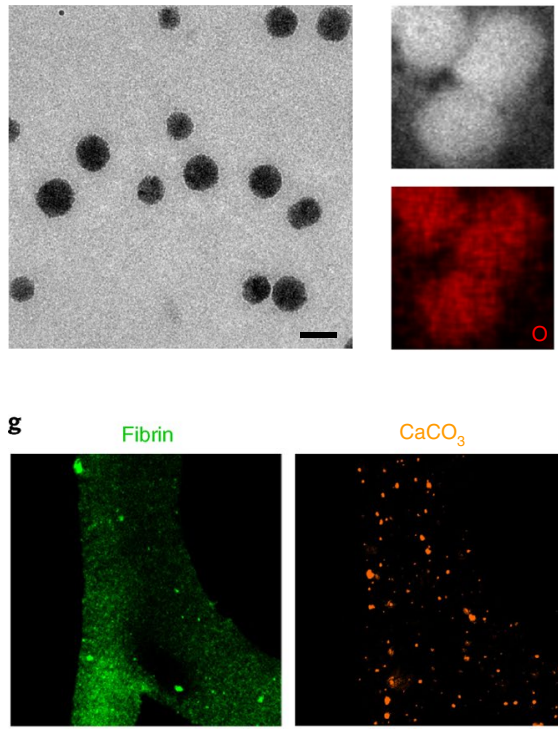

h

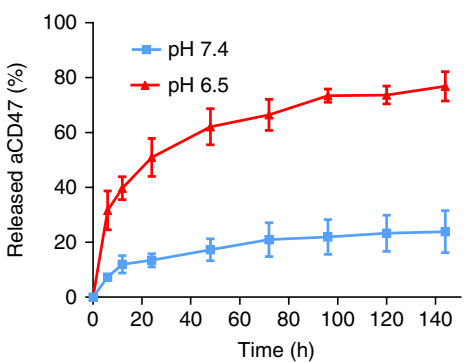

d
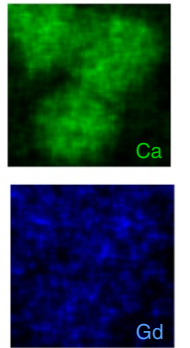

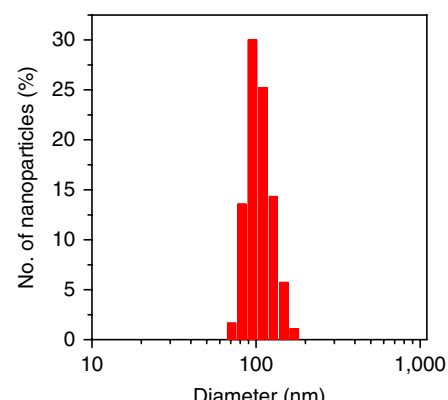

Diameter (nm)
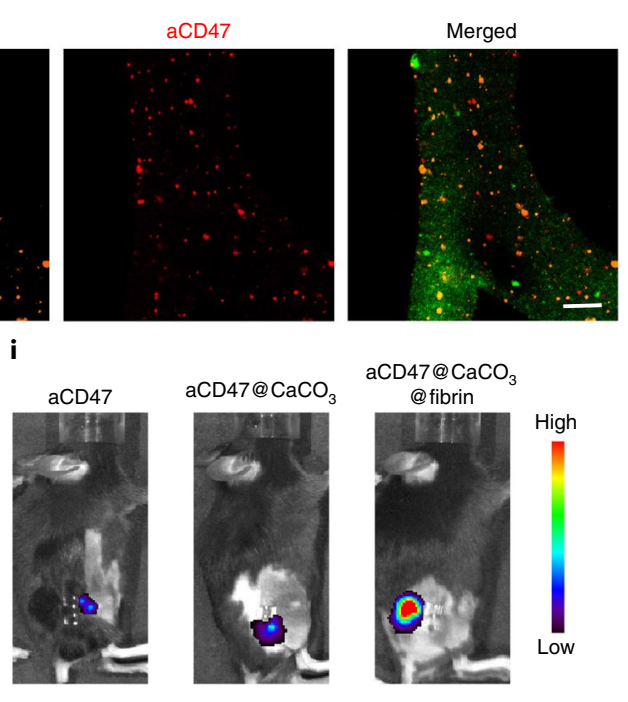

aCD47

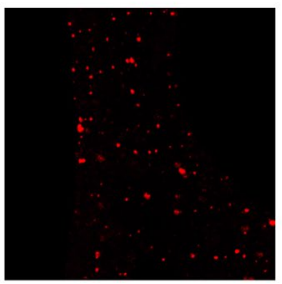

$\mathrm{aCD} 47 @ \mathrm{CaCO}_{3}$ $\underset{\text { aCD47@ fibrin }}{\text { CaCO }}$

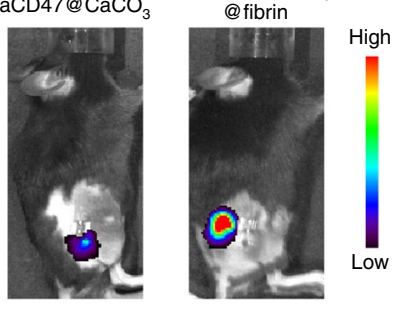

j

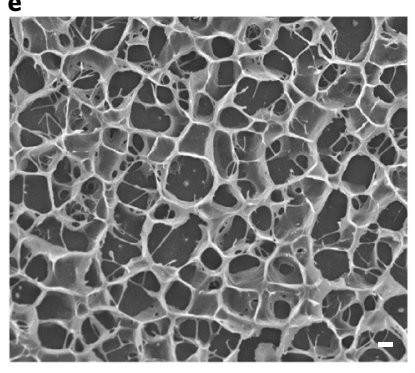

f

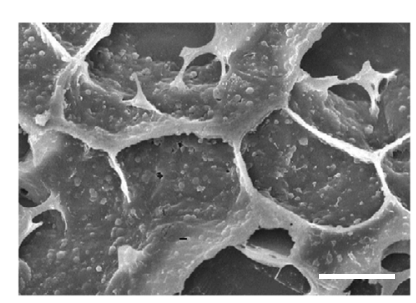

Fig. 1 | Schematic and characterization of the in situ formed immunotherapeutic fibrin gel. a, Schematic showing the in situ sprayed bioresponsive fibrin gel containing aCD47@ $\mathrm{CaCO}_{3}$ nanoparticles within the post-surgery tumour bed. aCD47@CaCO ${ }_{3}$ nanoparticles encapsulated in fibrin scavenge $\mathrm{H}^{+}$in the surgical wound site and release aCD47, thus promoting both polarization of TAMs to an M1-like phenotype and blockade of the 'don't eat me' signal in cancer cells. b. Transmission electron microscopy (TEM) image of aCD47@CaCO nanoparticles. Scale bar, 100 nm. Experiments were repeated three times; a representative image is shown. c, Respresentative scanning TEM images of aCD47@CaCO 3 nanoparticles showing the calcium (green), oxygen (red) and gadolinium labelled aCD47 (blue). Experiments were repeated three times. d, Average hydrodynamic size of aCD47@CaCO $\mathrm{CD}_{3}$ nanoparticles determined by dynamic light scattering. e,f, Representative cryo-scanning electron microscope (SEM) images of fibrin gel loaded with aCD47@CaCO 3 nanoparticles. Scale bars, $1 \mu$ m. Experiments were repeated three times. $\mathbf{g}$, Representative fluorescent images of a cryosection of fibrin gel, in which fibrinogen was labelled with $\mathrm{FITC}_{1} \mathrm{CaCO}_{3}$ nanoparticles with Cy5.5 (Cy5.5-labelled PEG-b-P(Glu) copolymers), and aCD47 was stained with rhodamine-conjugated anti-rat IgG antibody. Scale bar, $10 \mu \mathrm{m}$. Experiments were repeated three times. $\mathbf{h}$, Cumulative release profiles of aCD47 from fibrin in solutions at different $\mathrm{pH}$ values. Data are presented as mean \pm s.e.m. $(n=3)$. i, Fluorescence IVIS imaging depicting the in vivo retention of aCD47-Cy5.5, 6 days after delivery of antibodies in different formulations. Experiments were repeated three times. j, Quantification of the in vivo retention profile of aCD47-Cy5.5. FL, fluorescence; a.u., arbitrary unit. Data are presented as mean \pm s.e.m. $(n=3)$. Statistical significance was calculated via one-way analysis of variance (ANOVA) with a Tukey post-hoc test. ${ }^{\star} P<0.05$. 

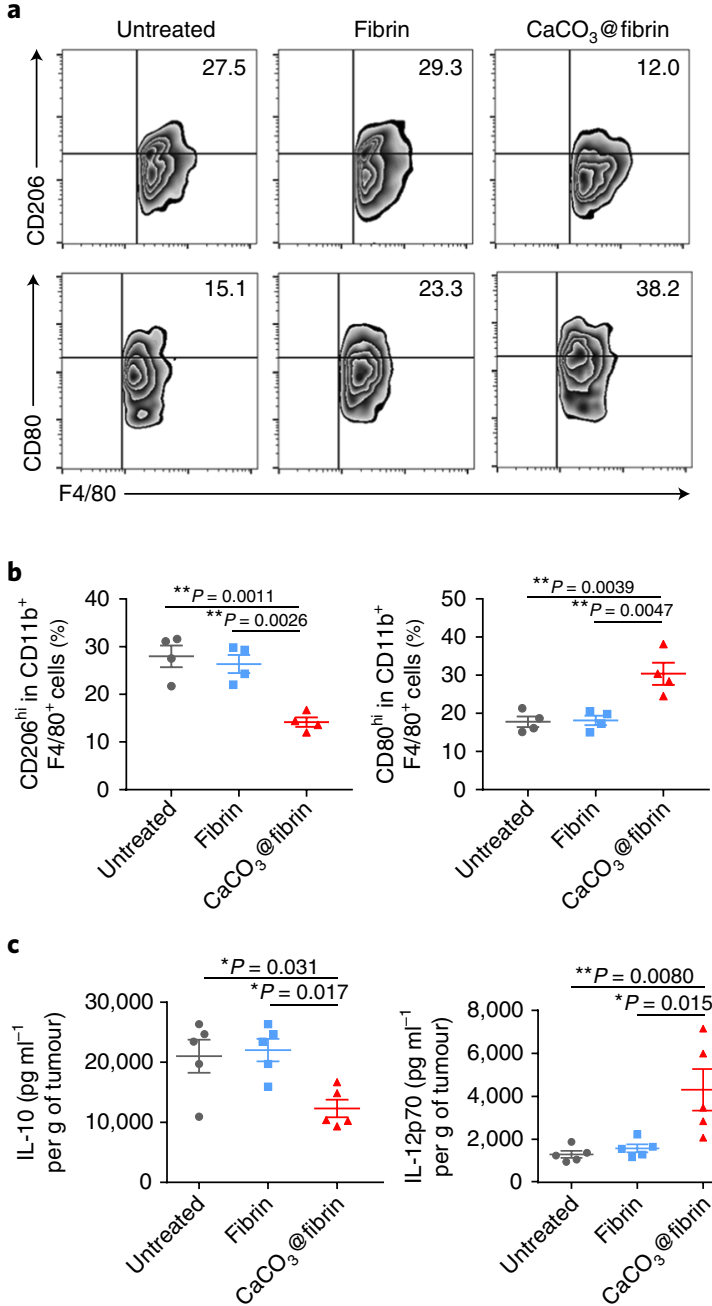

d

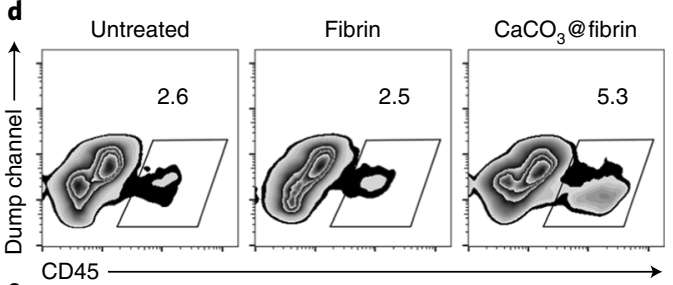

e
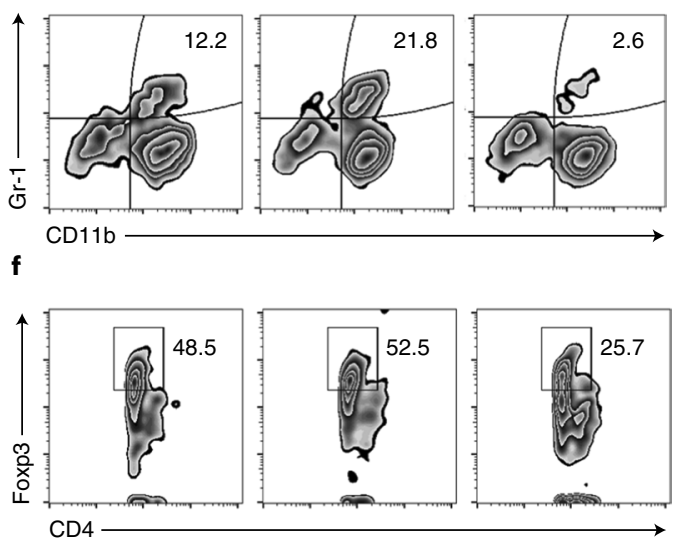

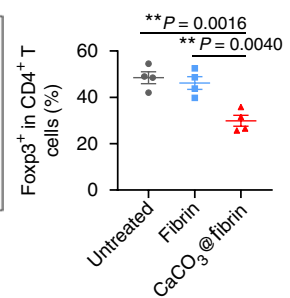

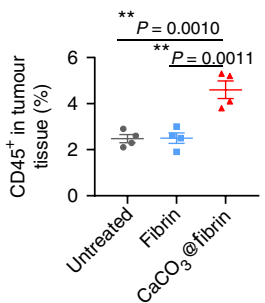

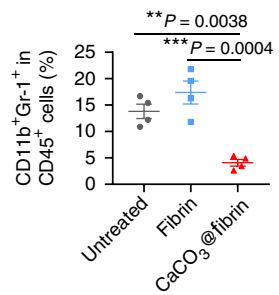

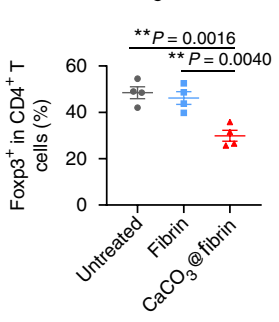

g
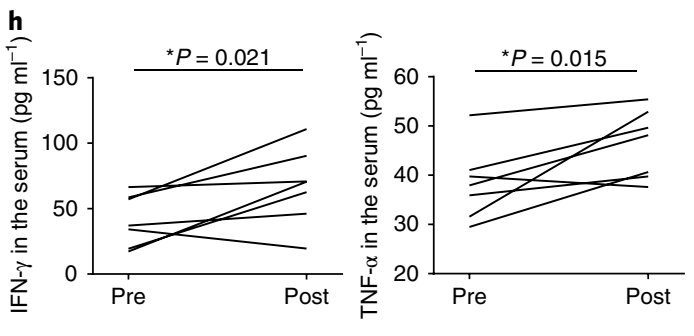
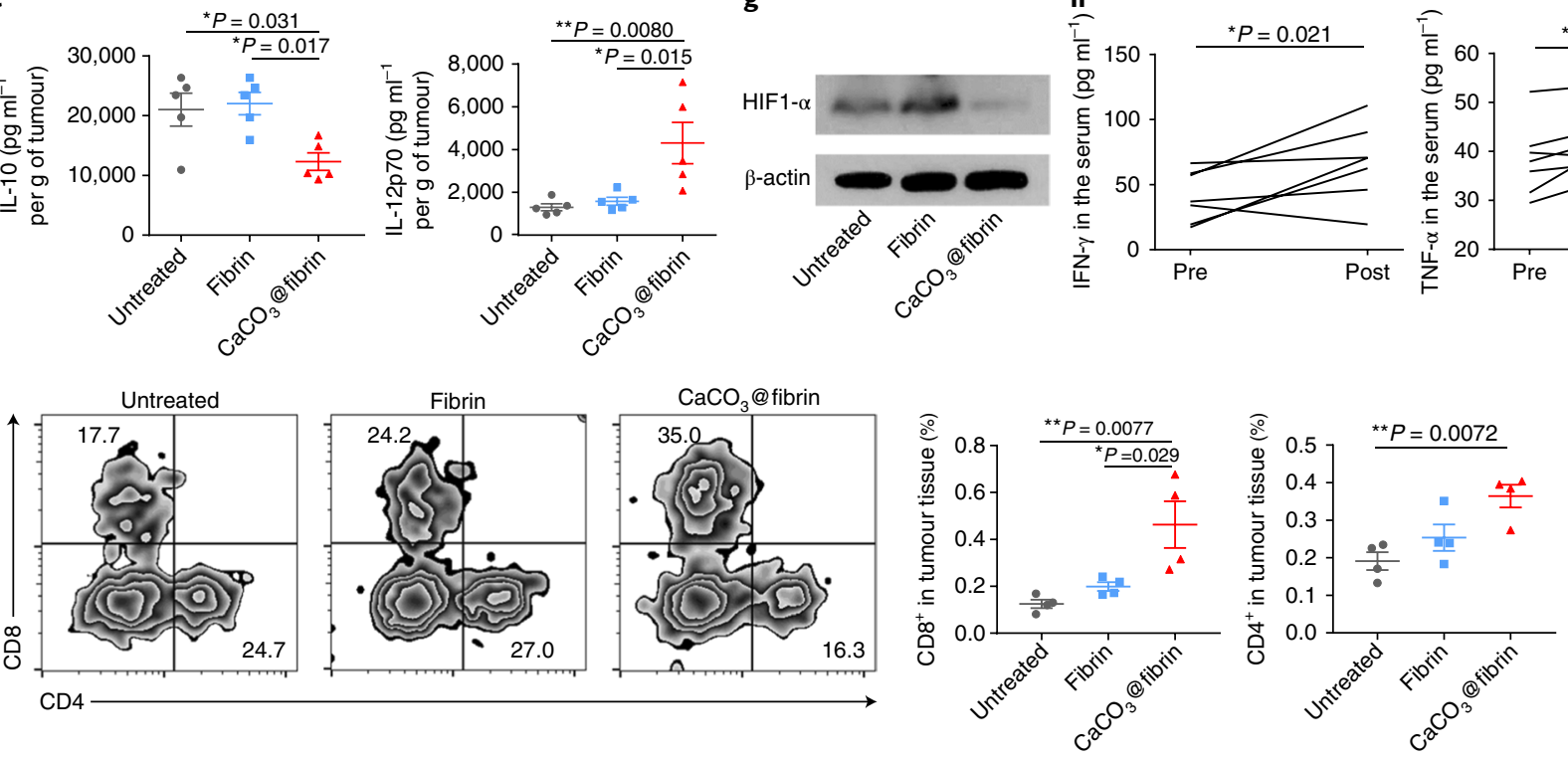

Fig. 2 | Incorporation of $\mathrm{CaCO}_{3} @$ fibrin for relieving immunosuppressive TME. B16F10 tumours were collected from mice 5 days after treatment.

$\mathbf{a}, \mathbf{b}$, Representative flow cytometric analysis images (a) and the relative quantification of M2-like macrophages (CD206 hi) and M1-like macrophages (CD80 hi) gating on $\mathrm{F} 4 / 80^{+} \mathrm{CD} 11 \mathrm{~b}^{+} \mathrm{CD} 45^{+}$cells $(\mathbf{b})$. Data are presented as mean \pm s.e.m. $(n=4)$. c, Secretion levels of IL-10 and IL-12p70 in different tumours. Data are presented as mean \pm s.e.m. $(n=5)$. d, Representative flow cytometric analysis images (left) and relative quantification (right) of CD45 $5^{+}$cells. Data are presented as mean \pm s.e.m. $(n=4)$. e, Representative flow cytometric analysis images (left) and relative quantification (right) of MDSCs $\left(C D 11 b^{+} G r-1^{+}\right)$gating on CD45 $5^{+}$ cells. Data are presented as mean \pm s.e.m. $(n=4)$. f, Representative flow cytometric analysis images (left) and relative quantification (right) of CD4+Foxp3 ${ }^{+} T$ cells gating on $\mathrm{CD}^{+}{ }^{+} \mathrm{CD} 4^{+}$cells. Data are presented as mean \pm s.e.m. $(n=4), \mathbf{g}$, HIF1- $\alpha$ protein expression levels in B16F10 tumours analysed by western blotting. Experiments were repeated three times. $\mathbf{h}$, Systemic IFN- $\gamma$ and TNF- $\alpha$ levels before and after $\mathrm{CaCO}_{3} @$ fibrin treatment $(n=7)$. i, Representative flow cytometric analysis gating on $\mathrm{CD}^{+}$cells (left) and absolute quantification (right) of $\mathrm{CD} 8^{+}$and $\mathrm{CD} 4^{+} \mathrm{T}$ cells in the tumour. Data are presented as mean \pm s.e.m. $(n=4)$. Statistical significance was calculated via one-way ANOVA with a Tukey post-hoc test $(\mathbf{b}, \mathbf{c}, \mathbf{d}-\mathbf{f}, \mathbf{i})$ or Student's $t$-test $(\mathbf{h}) .{ }^{\star} P<0.05 ;{ }^{* \star} P<0.01 ;{ }^{\star \star \star} P<0.001$.

antitumour T cell responses, while reducing the toxic effects associated with the systemic administration of aCD47.

\section{In situ formation of fibrin gel by spray}

aCD47@ $\mathrm{CaCO}_{3}$ nanoparticles with a loading capacity of 5\% and encapsulation efficiency of $50 \%$ were prepared by precipitation of
$\mathrm{Ca}^{2+}$ and $\mathrm{CO}_{3}{ }^{2-}$ in a solution containing poly(ethylene glycol)-bpoly(glutamic acid) (PEG-b-P(Glu)) block copolymers according to the methods in a previous study ${ }^{37}$. Monodisperse $\mathrm{aCD}^{2}$ @ $\mathrm{CaCO}_{3}$ nanoparticles with a -diameter of $\sim 100 \mathrm{~nm}$ were obtained, the size of which was controlled by the $\mathrm{P}(\mathrm{Glu})$ block through the interaction between carboxyl and $\mathrm{Ca}^{2+}$, as well as a PEG shell to avoid 
a

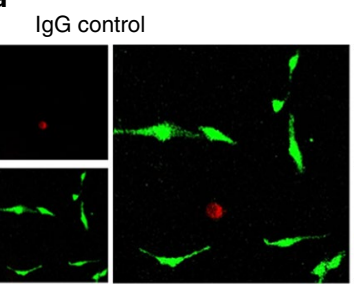

aCD47

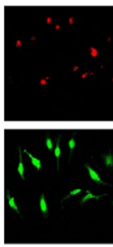

b

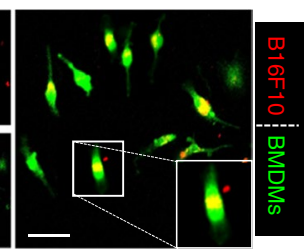

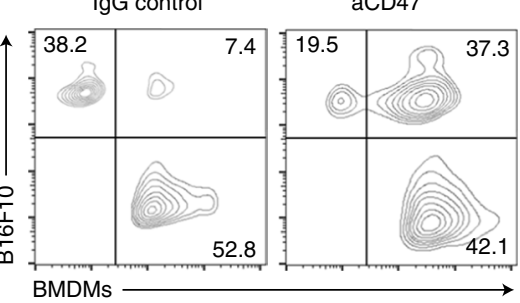

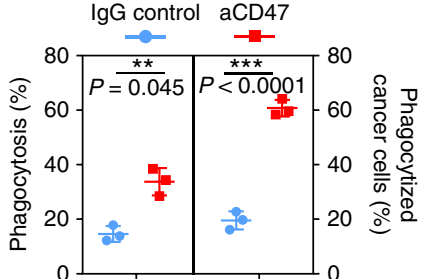

e
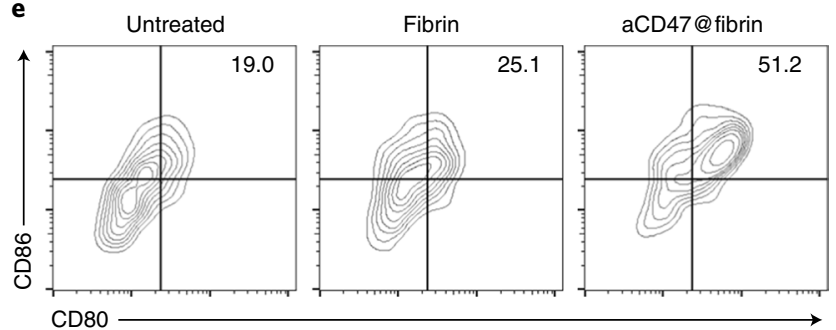
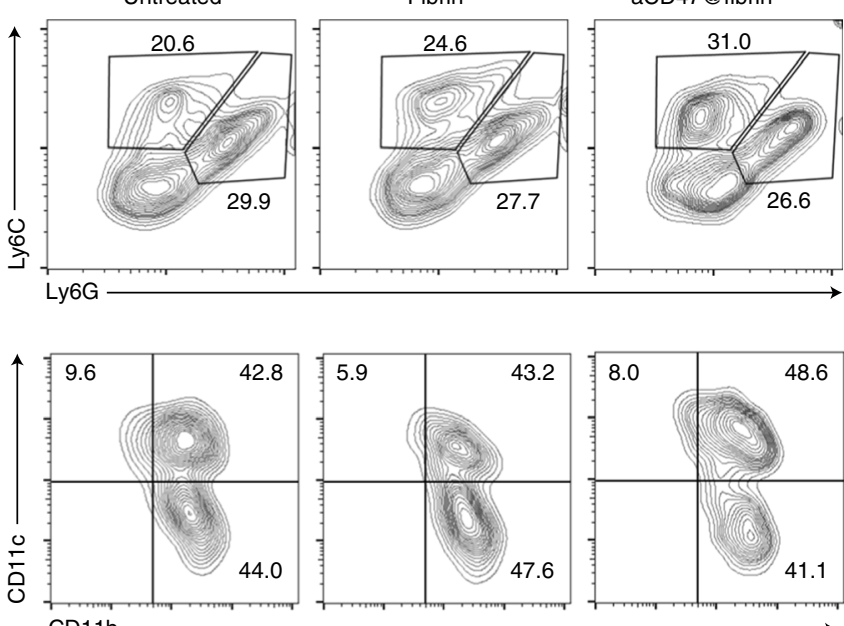

CD11b
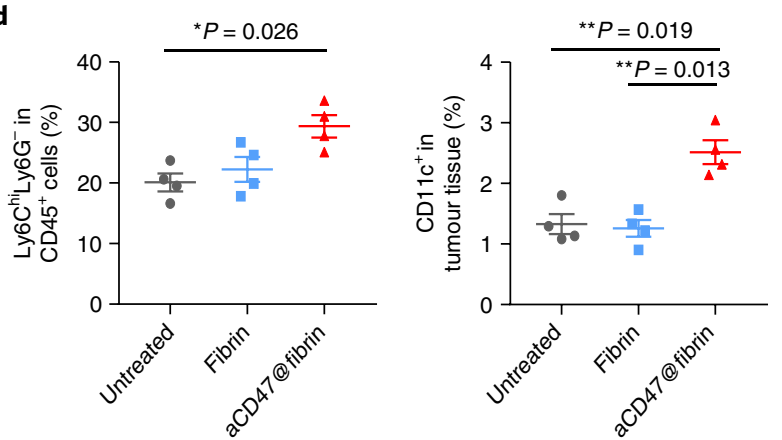

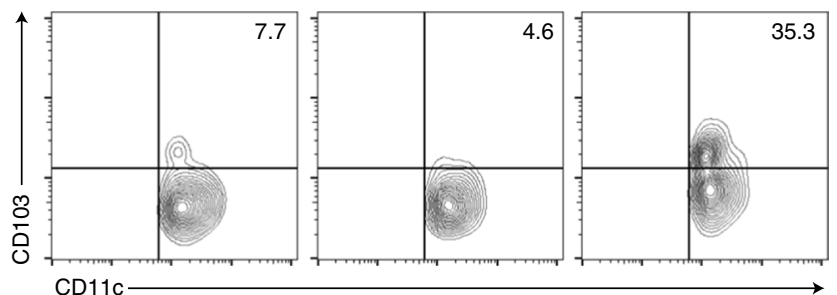

$\mathbf{f}$
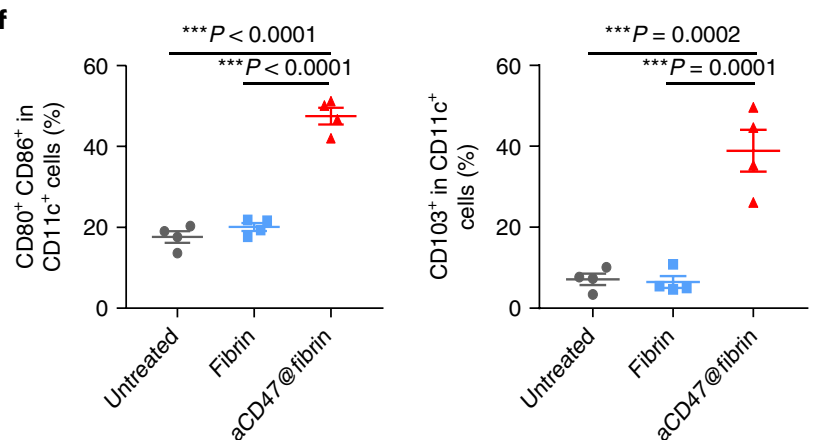

Fig. 3 | CD47 blockade for increasing phagocytosis in vitro and exerting antitumour immune responses in vivo. a, Representative confocal images of phagocytosis assays, in which B16F10 cancer cells were labelled with CellTracker DeepRed (red) and bone-marrow-derived macrophage cells (BMDMs) were labelled with CellTracker Green (green). Scale bar, $50 \mu \mathrm{m}$. Experiments were repeated three times. b, Representative flow cytometric analysis images (left) and relative quantification (right) of the phagocytosis of cancer cells by BMDMs. Data are presented as mean \pm s.e.m. ( $n=3$ ). Phagocytosis or phagocytized cancer cells were quantified as the percentage of double-positive BMDMs among CellTracker Green-positive BMDMs or CellTracker DeepRed-cancer cells. c,d, Representative flow cytometric analysis images (c) and relative quantification of Ly6ChiLy6G- macrophages gating on CD45+ cells and the absolute percentage of $\mathrm{CD}_{11 c^{+}}$cells in the tumour $(\mathbf{d})$. Experiments were repeated three times. Data are presented as mean $\pm \mathrm{s} . \mathrm{e} . \mathrm{m}$. $(n=4)$. e,f, Representative flow cytometric analysis images (e) and relative quantification of CD80+CD86 ${ }^{+}$dendritic cells and CD103+ dendritic cells gating on $\mathrm{CD} 45^{+} \mathrm{CD} 11 \mathrm{c}^{+}$cells (f). Data are presented as mean \pm s.e.m. $(n=4)$. Statistical significance was calculated via two-tailed Student's $t$-test $(\mathbf{b})$ or one-way ANOVA with a Tukey post-hoc test $(\mathbf{d}, \mathbf{f}) .{ }^{\star} P<0.05 ;{ }^{\star \star} P<0.01 ;{ }^{\star \star \star} P<0.001$.

further aggregation and agglomeration(Fig. 1b-d). Elemental mapping images (Fig. 1c) indicated the homogeneous distribution of aCD47 (gadolinium-chelated) in the $\mathrm{CaCO}_{3}$ nanoparticles.

Fibrin gel was quickly formed by simultaneously spraying and mixing equal volumes of solutions containing either fibrinogen with size-optimized aCD47@ $\mathrm{CaCO}_{3}$ nanoparticles or thrombin, which was validated by a rheology test (Supplementary Fig. 1). $\mathrm{Ca}^{2+}$ associated with $\mathrm{CaCO}_{3}$ nanoparticles in the solution facilitates the formation of fibrin gel ${ }^{38}$. The mixture of the two solutions resulted in a rapid increase in the elastic modulus $\left(G^{\prime}\right)$. The morphology of the fibrin gel containing aCD47@ $\mathrm{CaCO}_{3}$ nanoparticles was observed using cryo-scanning electron microscopy (cryo-
SEM) imaging (Fig. 1e,f). To further substantiate the distribution of aCD47@ $\mathrm{CaCO}_{3}$ nanoparticles in the hydrogel, fluorescein (FITC)-labelled fibrinogen and Cy5.5-labelled $\mathrm{CaCO}_{3}$ nanoparticles were used for hydrogel formation. Frozen sections were stained with rhodamine-conjugated donkey anti-rat IgG antibody to detect the aCD47. As demonstrated by confocal imaging, the Cy5.5 signals from $\mathrm{CaCO}_{3}$ exhibited a uniform distribution pattern in the gel and were co-localized with aCD47, further indicating the encapsulation of aCD47 in the $\mathrm{CaCO}_{3}$ nanoparticles (Fig. 1g). The biodegradation behaviour of the Cy5.5-labelled fibrin gel was monitored using a fluorescence in vivo imaging system (IVIS). Three weeks after spraying, the fluorescence signal 
a

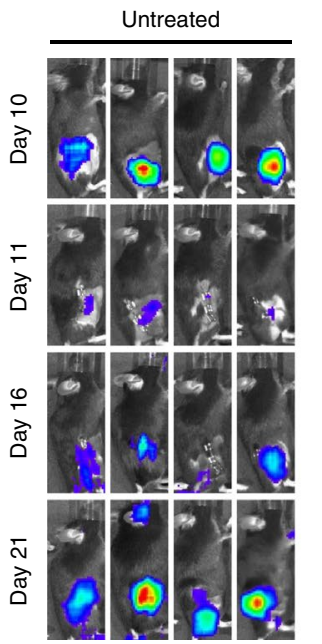

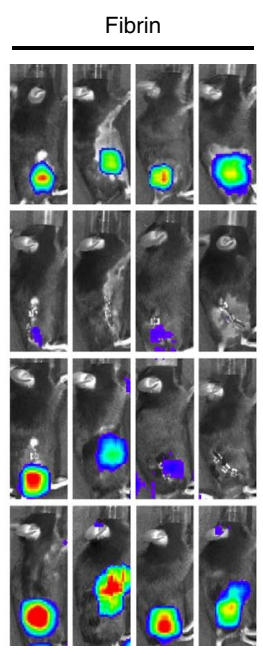

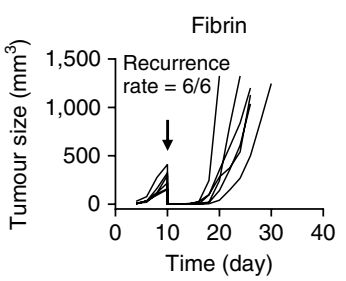

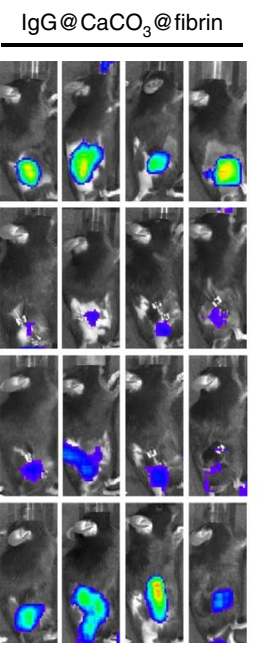
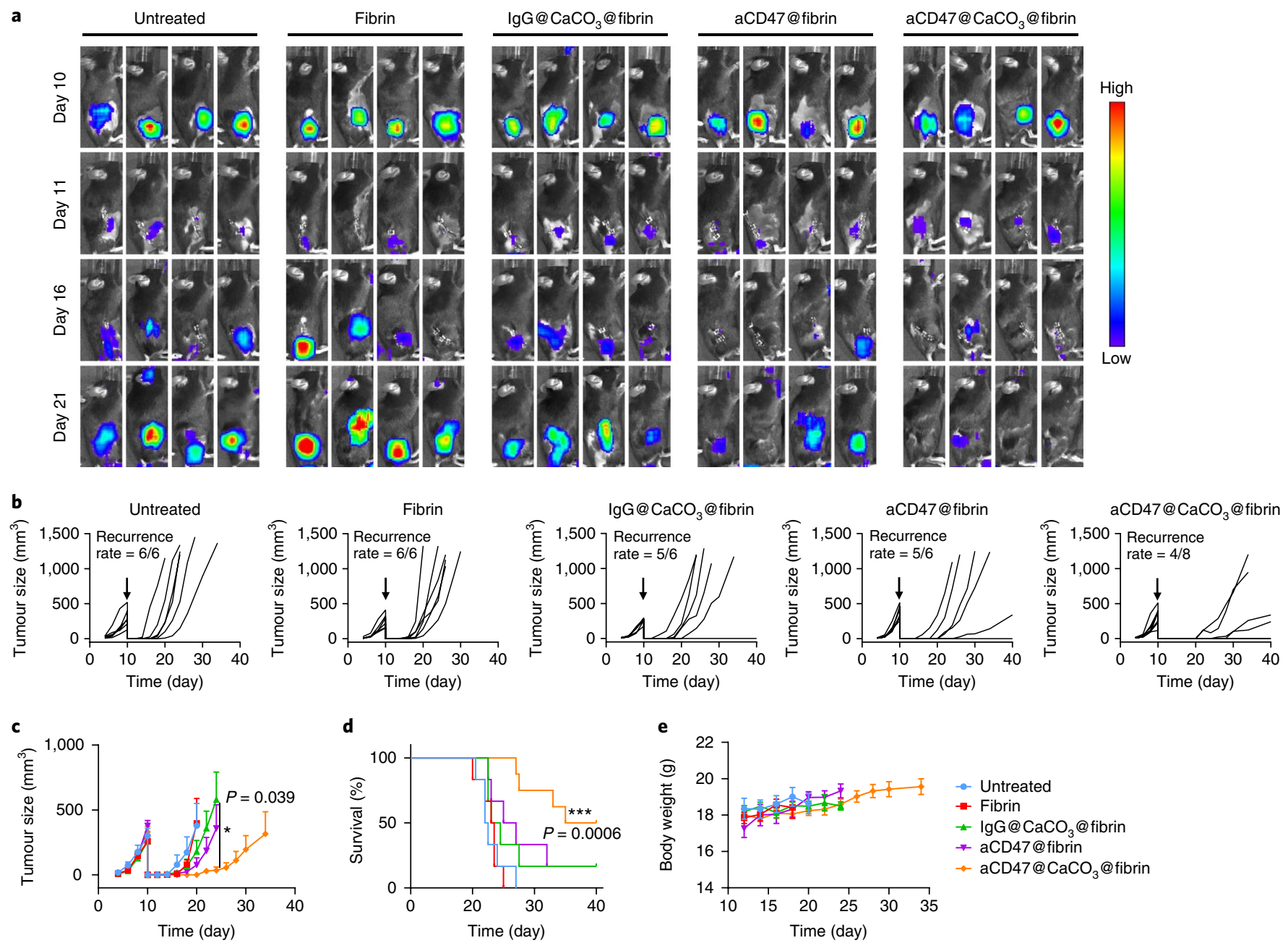

Fig. 4 | aCD47@CaCO $@$ @fibrin for reducing recurrence of B16F10 tumours after surgery. a, In vivo bioluminescence imaging of B16F10 tumours after removal of the primary tumour. Four representative mice per treatment group are shown. Images associated with day 10 were taken before surgery. Experiments were repeated three times. b,c, Individual (b) and average (c) tumour growth kinetics in different groups. Growth curves were stopped when the first mouse of the corresponding group died. Data are presented as mean \pm s.e.m. $(n=8$ for the aCD47@CaCO $3 @$ fibrin-treated group, $n=6$ for the other groups). Statistical significance was calculated via one-way ANOVA with a Tukey post-hoc test for multiple comparisons. d, Survival corresponding to the tumour size of mice after different treatments as indicated ( $n=8$ for the $\mathrm{CCD} 47 @ \mathrm{CaCO}_{3} @$ fibrin-treated group, $n=6$ for the other groups). Statistical significance was calculated via the log-rank (Mantel-Cox) test. e, Weight changes of mice in different groups. Data are presented as mean \pm s.e.m. ( $n=8$ for the aCD47@CaCO $@$ fibrin-treated group, $n=6$ for the other groups). ${ }^{\star} P<0.05,{ }^{\star \star \star} P<0.001$.

from the gel was undetectable, indicating degradation of the gel (Supplementary Fig. 2).

The $\mathrm{CaCO}_{3}$ nanoparticles dissolved and released the encapsulated $\mathrm{aCD} 47$ by reacting with $\mathrm{H}^{+}$in the acidic buffer (Supplementary Figs. 3 and 4). Meanwhile, $\mathrm{CaCO}_{3}$ nanoparticles elevated the $\mathrm{pH}$ values of the acidic solution as a proton scavenger (Supplementary Fig. 5). Encapsulation of aCD47@ $\mathrm{CaCO}_{3}$ nanoparticles in the hydrogel allowed a gradual release of aCD47 (Fig. 1h). To evaluate the release behaviour in vivo, aCD47 was either dispersed in phosphate buffered saline (PBS), encapsulated in $\mathrm{CaCO}_{3}$ nanoparticles or in $\mathrm{CaCO}_{3} @$ fibrin gel, and then injected or sprayed into the tumour resection cavity. The Cy5.5-labelled aCD47 signal was monitored and quantified by an IVIS (Fig. 1i,j and Supplementary Fig. 6). While $80 \%$ of the signal from the injected antibody in solution was undetectable at the resection site, $79 \%$ of aCD47 released from the $\mathrm{CaCO}_{3} @$ fibrin gel remained detectable. The sustained release of aCD47 was further substantiated by confocal imaging of residual tumour sections, in which a remarkable fluorescence signal associated with the antibodies was observed away from the nanoparticles' signal, indicating degradation of the $\mathrm{CaCO}_{3}$ nanoparticles and sustained release of antibodies from the nanoparticles (Supplementary Fig. 7). Although the sustained release of therapeutics from the gelnanoparticle reservoir is advantageous compared with free cargo, the release kinetics for this application could be further optimized to maximize the therapeutic effects and minimize toxicities.

\section{Immune response induced by $\mathrm{CaCO}_{3}$ and $\mathrm{aCD} 47$}

Glycolytic metabolism of cancer cells in the hypoxic TME leads to the production of lactate and $\mathrm{H}^{+}(\mathrm{pH} 6.5-6.8)$, both of which impair the functions of immune cells $\mathrm{s}^{39}$. Local acidification ( $\left.\mathrm{pH} 6.0-7.0\right)$ is also observed in inflamed and injured tissues ${ }^{36}$. Considering the ability of $\mathrm{CaCO}_{3}$ to scavenge $\mathrm{H}^{+}$, we examined the immune effects caused by spreading of the $\mathrm{CaCO}_{3} @$ fibrin gel $\left(1 \mathrm{mg} \mathrm{CaCO}_{3}\right.$ per mouse) within the tumour resection cavity. We observed a reduction of M2-like TAMs (CD206 $\left.{ }^{\mathrm{hi}} \mathrm{CD} 11 \mathrm{~b}^{+} \mathrm{F} 4 / 80^{+}\right)$and an increase of M1-like TAMs $\left(\mathrm{CD} 80^{\text {hi }} \mathrm{CD} 11 \mathrm{~b}^{+} \mathrm{F} 4 / 80^{+}\right)$(Fig. 2a,b) ${ }^{40,41}$. This polarization was further confirmed by the reduced level of IL-10 (the predominant cytokine secreted by M2 macrophages) and the increased 

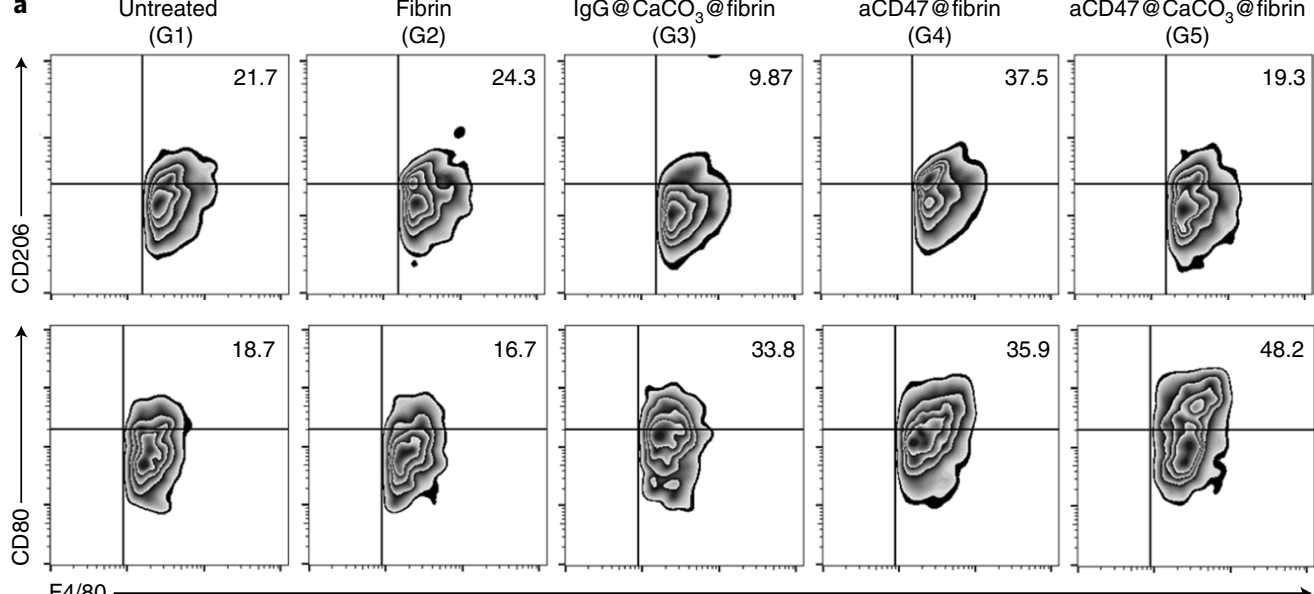

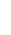
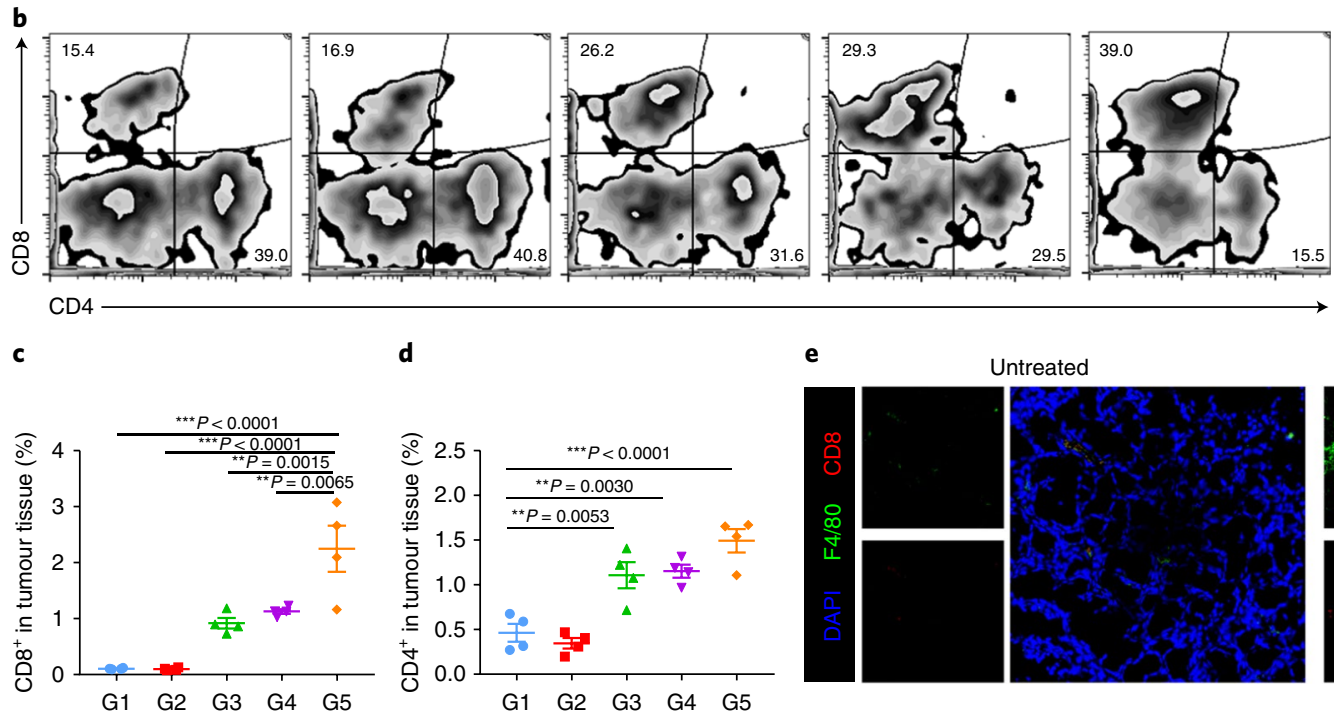

d

e

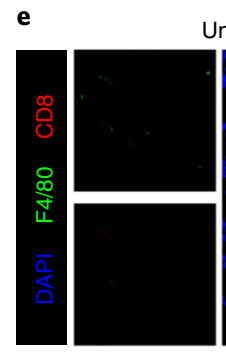

Untreated
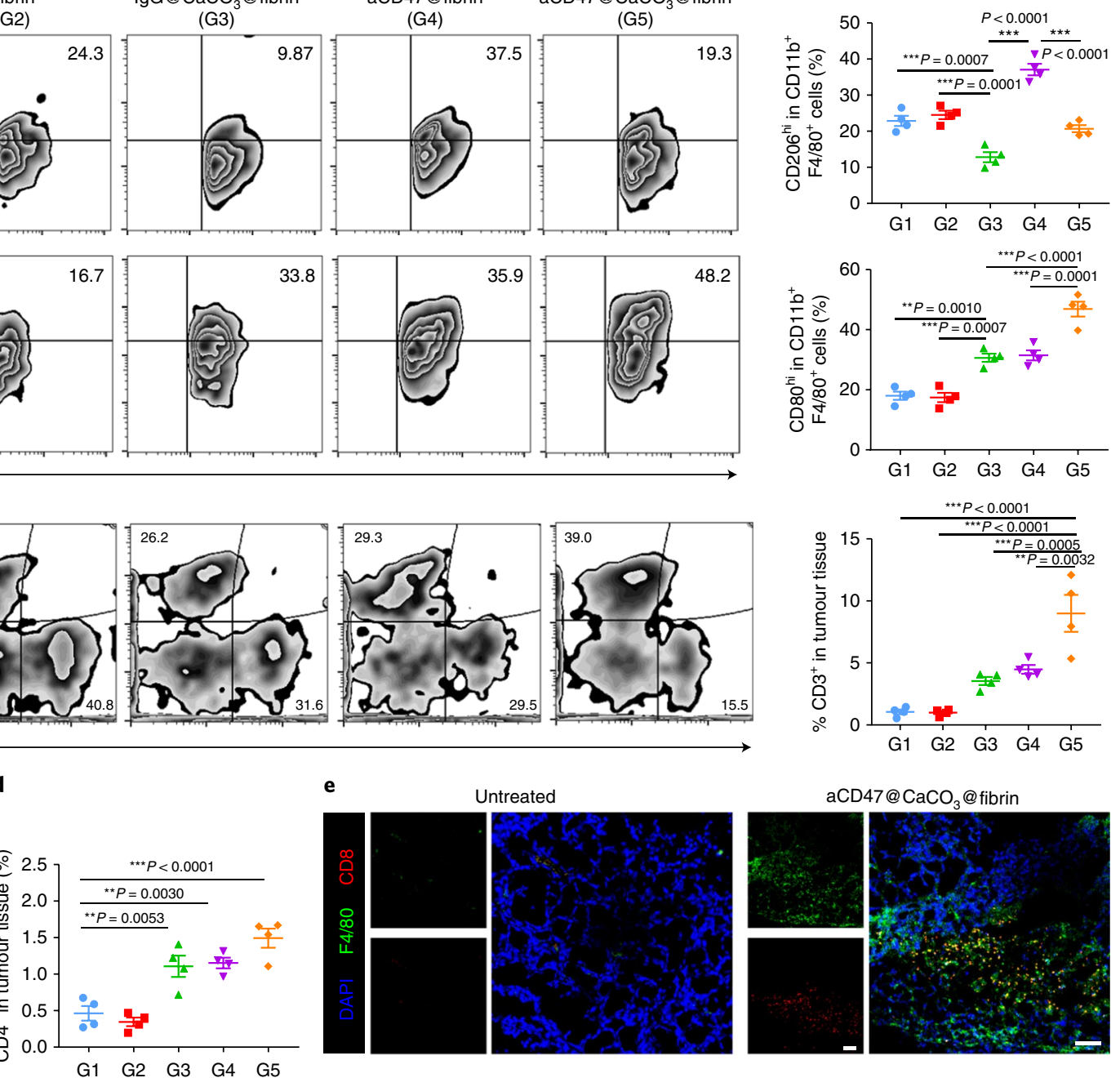

aCD47@ $\mathrm{CaCO}_{3} @$ fibrin
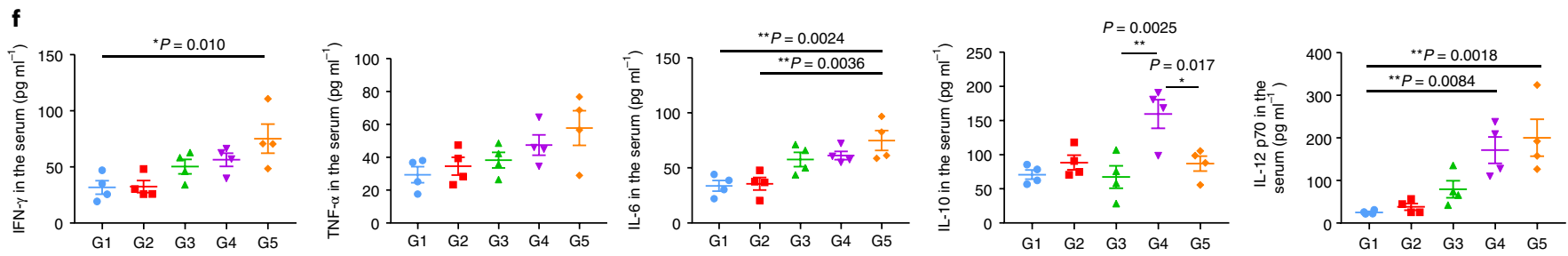

Fig. 5 | aCD47@ $\mathrm{CaCO}_{3} @$ fibrin for triggering antitumour immune response. B16F10 tumours were harvested from mice 5 days after treatment. a, Representative flow cytometric analysis images (left) and relative quantification (right) of M2-like macrophages (CD206 ${ }^{\text {hi }}$ ) and M1-like macrophages $\left(C D 80^{\text {hi }}\right)$ gating on $\mathrm{F} 4 / 80^{+} \mathrm{CD} 11 \mathrm{~b}^{+} \mathrm{CD} 45^{+}$cells. Data are presented as mean \pm s.e.m. $(n=4)$. $\mathbf{b}$, Representative flow cytometric analysis of T cell infiltration within the tumour gating on $\mathrm{CD}^{+}$cells (left) and the quantification results (right) in different groups. Data are presented as mean $\pm \mathrm{s} . \mathrm{e} . \mathrm{m}$. ( $n=4$ ). $\mathbf{c}, \mathbf{d}$, Absolute percentage of the $\mathrm{CD}^{+}(\mathbf{c})$ and $\mathrm{CD}^{+}(\mathbf{d}) \mathrm{T}$ cells within tumours following various treatments. Data are presented as mean $\pm \mathrm{s} . \mathrm{e} . \mathrm{m}$. $(n=4)$.

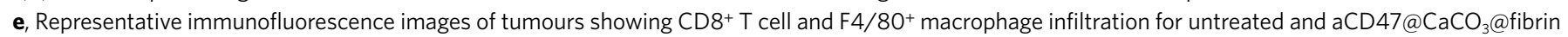
groups. Scale bars, $50 \mu \mathrm{m}$. Experiments were repeated three times. f, Cytokine levels in the serum from mice isolated 5 days after different treatments. Data are presented as mean \pm s.e.m. $(n=4)$. G1, untreated; G2, fibrin; G3, IgG@CaCO $@$ fibrin; G4, aCD47@fibrin; G5, aCD47@CaCO_@fibrin. Statistical significance was calculated via one-way ANOVA with a Tukey post-hoc test. ${ }^{\star} P<0.05 ;{ }^{\star \star} P<0.01 ;{ }^{\star \star \star} P<0.001$.

level of interleukin-12 (IL-12, the predominant cytokine secreted by M1 macrophages) in the TME (Fig. 2c). The observed polarization of macrophages to the M1 phenotype could be ascribed to modulation of the acidity of the TME, which usually favours M2-like macrophage polarization ${ }^{40}$. Compared to small molecules that promote M1-like macrophage polarization ${ }^{42}$, the $\mathrm{CaCO}_{3}$ scavenging of $\mathrm{H}^{+}$within the inflamed tumour resection provides a simple approach. A correlation was observed between the concentration of $\mathrm{CaCO}_{3}$ nanoparticles and macrophage polarization, as assessed by flow cytometry (Supplementary Fig. 8). After $\mathrm{CaCO}_{3} @ f i b r i n$ treatment, reduction of myeloid-derived suppressor cells (MDSCs) and regulatory $\mathrm{T}$ cells (Tregs) and reduced expression of hypoxiainducible factor 1- $a$ (HIF1- $a$ ) in the TME were also observed (Fig. 2d-g and Supplementary Fig. 9). Remarkably, $\mathrm{CaCO}_{3} @$ fibrin treatment caused an increase in tumour-infiltrating lymphocytes (TILs, $\mathrm{CD}^{+}$), especially cytotoxic T lymphocytes (CTLs, 
a
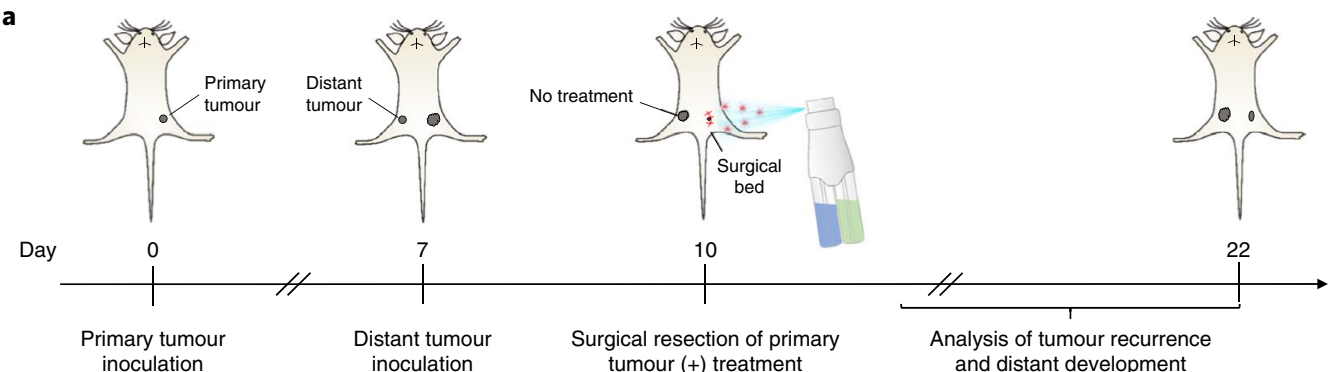

b
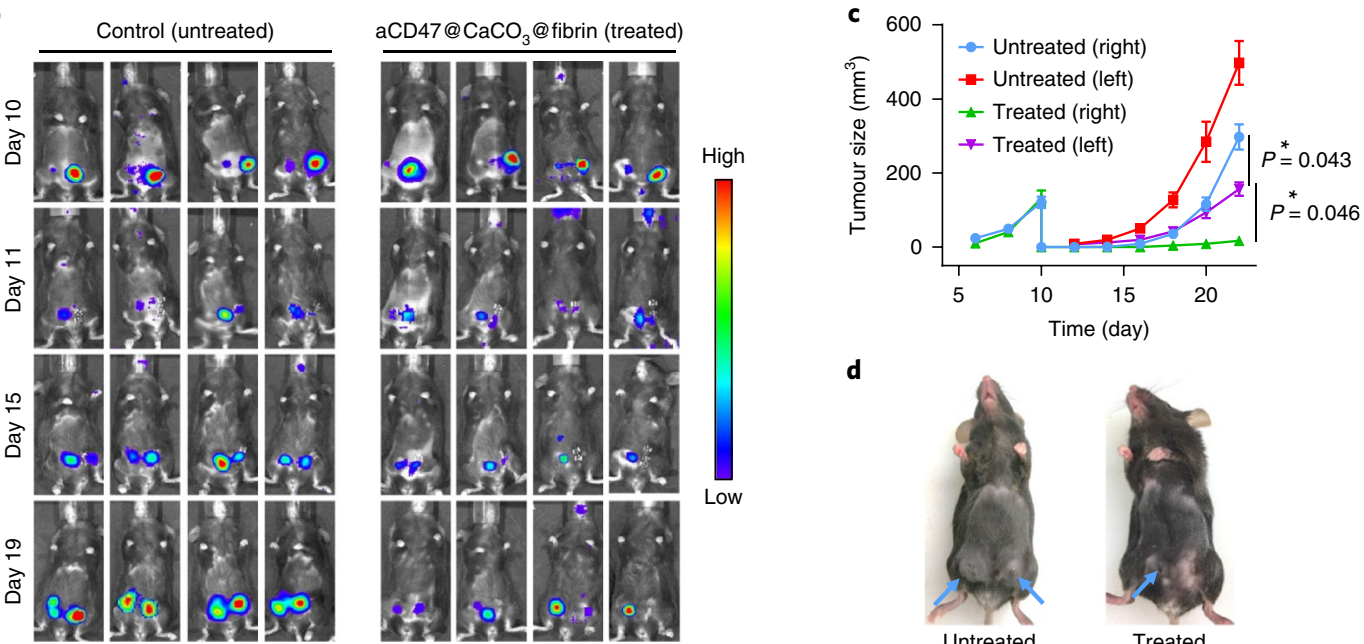

d
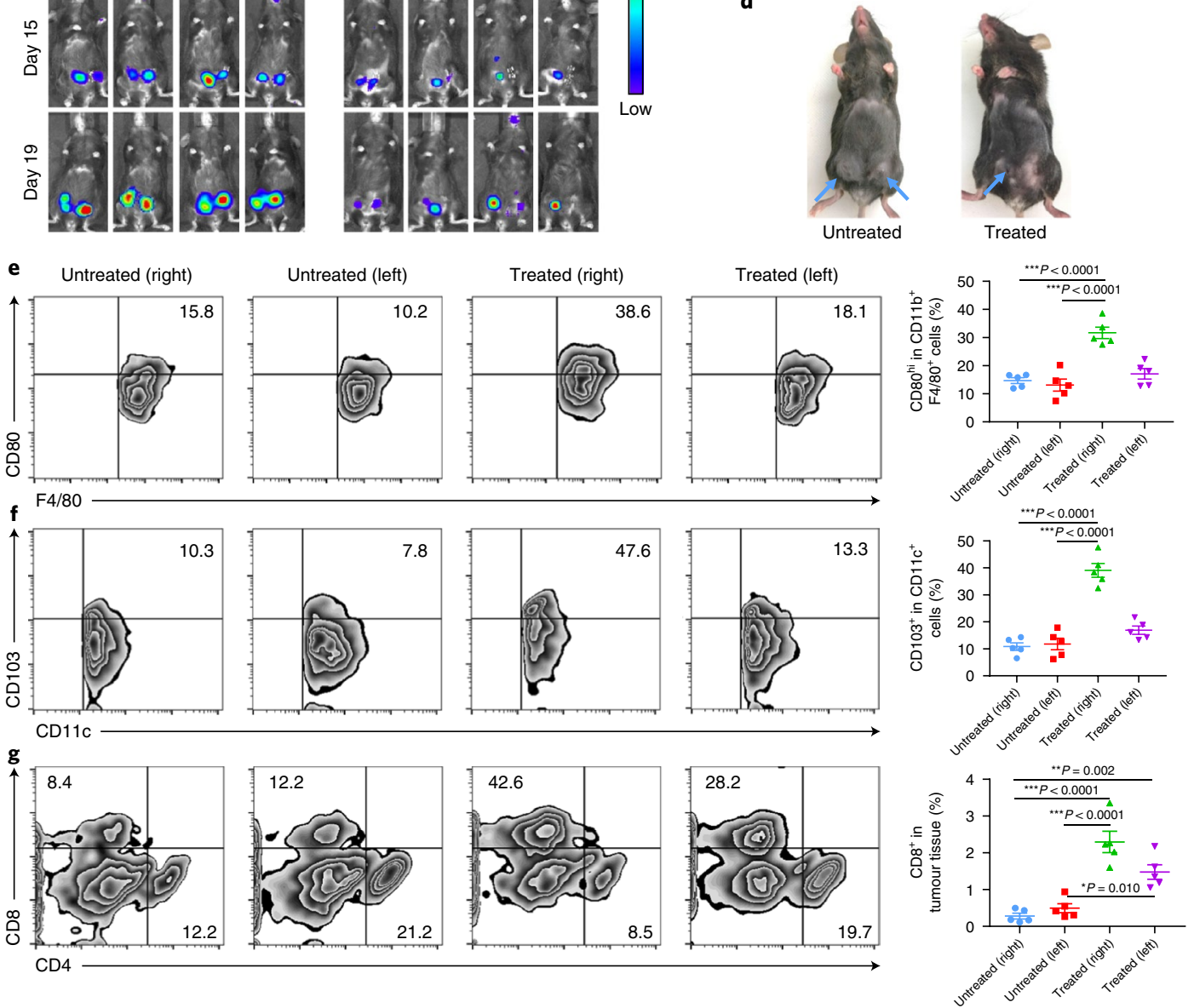

Fig. 6 | Local treatment of aCD47@CaCO 3 @fibrin for systemic antitumour immune response. a, Schematic illustrating aCD47@CaCO $@$ @fibrin therapy in a mouse model of incomplete tumour resection and distant tumour.Tumours on the right side were designated as 'primary tumours' with aCD47@CaCO @ fibrin treatment, and those on the left side were designated as 'distant tumours' without any treatment. $\mathbf{b}$, In vivo bioluminescence imaging of B16F10 tumours in response to local aCD47@ $\mathrm{CaCO}_{3} @$ fibrin treatment. Images associated with day 10 were taken before surgery. Experiments were repeated three times. c, Growth curves for left and right tumours in untreated and treated mice. Data are presented as mean \pm s.e.m. $(n=8)$. d, Representative mice photographs at day 22. Blue arrows indicate tumours. Experiments were repeated three times. e, Representative flow cytometric analysis images (left) and relative quantification (right) of M1-like macrophages $\left(C D 80^{\text {hi }}\right)$ gating on F4/80+CD11 b+CD45+ cells. Data are presented as mean \pm s.e.m. $(n=5)$. f, Representative flow cytometric analysis images (left) and relative quantification (right) of $C D 103^{+}$dendritic cells gating on $C D 45^{+} C D 11 c^{+}$cells. Data are presented as mean \pm s.e.m. $(n=5)$. $\mathbf{g}$, Representative flow cytometric analysis of T cell infiltration gating on $\mathrm{CD}^{+}$cells within the tumour (left) and the absolute percentage (right) of CD8 ${ }^{+}$T cells in the tumour in different groups. Data are presented as mean \pm s.e.m. $(n=5)$. Experiments were repeated three times. Statistical significance was calculated via one-way ANOVA with a Tukey post-hoc test. ${ }^{\star} P<0.05 ;{ }^{\star \star} P<0.01 ;{ }^{\star \star \star} P<0.001$. 
$\mathrm{CD}^{+} \mathrm{CD}^{+}$), within the tumour (Fig. 2i), as well as an increase in interferon- $\gamma$ (IFN- $\gamma)$ and tumour necrosis factor- $\alpha$ (TNF- $\alpha)$ in the plasma (Fig. 2h). Meanwhile, the expression of programmed death-1 (PD-1) protein and programmed death-ligand 1 (PD-L1) on immune cells and cancer cells, respectively, remained nearly unchanged or slightly decreased (Supplementary Fig. 10).

CD47 blockade increased the phagocytosis of cancer cells by macrophages in vitro, as shown in the confocal imaging and flow cytometry results (Fig. 3a,b and Supplementary Fig. 11). When aCD47 was loaded into the fibrin gel $(50 \mu \mathrm{g}$ aCD47 per mouse) and sprayed at the tumour resection site, an increased frequency of Ly6C ${ }^{\text {hi }}$ Ly $6 \mathrm{G}^{-}$macrophages within the resection tumour cavity was observed, but not for Ly6 $\mathrm{G}^{+} \mathrm{Ly} 6 \mathrm{C}^{\mathrm{dim}}$ neutrophils (Fig. 3c,d and Supplementary Fig. 12). An increase in $\mathrm{CD} 11 \mathrm{c}^{+}$dendritic cells was also recorded, and these cells showed expression of CD80, CD86 and CD103, denoting their maturation status ${ }^{43}$ (Fig. 3e,f). Thus, CD47 blockade enhances phagocytosis of cancer cells by both macrophages and dendritic cells, activating innate immune systems.

Immunotherapeutic gel for inhibition of tumour recurrence To validate the therapeutic effects of aCD47@CaCO $3 @ f i b r i n, w e$ used an incomplete tumour resection model. The in situ formed fibrin gels containing IgG@CaCO$, \mathrm{CCD}_{3}, \mathrm{Cr}$ oCD47@ $\mathrm{CaCO}_{3}(1 \mathrm{mg}$ $\mathrm{CaCO}_{3}$ per mouse; $50 \mu \mathrm{g}$ aCD47 per mouse) were sprayed into the tumour resection cavity. Tumour growth was then monitored by bioluminescence signals from B16F10 cancer cells (Fig. 4a). Mice treated with the aCD47@CaCO $3 @$ fibrin showed improved control of tumour regrowth as four out of eight mice had no detectable tumour (Fig. 4b,c). Fifty per cent of the mice after aCD47@ $\mathrm{CaCO}_{3} @$ fibrin treatment survived for at least 60 days (Fig. 4d), and the body weights of mice were not impacted by the treatment (Fig. 4e). Moreover, histology analysis of major organs collected from mice 30 days after aCD47@CaCO 3 fibrin treatment, together with the complete blood panel test and serum biochemistry assay conducted at 1, 7 and 14 days after treatment, indicated that local delivery of aCD47 did not induce significant side effects to mice (Supplementary Figs. 13 and 14).

Residual tumours were harvested and analysed using flow cytometry and immunofluorescence staining 5 days after surgery. The proportion of both M1- and M2-type macrophages in the residual tumour was increased after aCD47 blockade, together with an increased polarization of M1-phenotype TAMs after $\mathrm{CaCO}_{3}$ treatment (Fig. 5a and Supplementary Fig. 15). Similarly, the absolute number of TILs $\left(\mathrm{CD}^{+}\right.$cells) was increased in the residual tumour

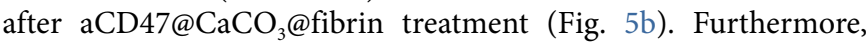
the percentage of $\mathrm{CD} 8^{+} \mathrm{T}$ cells was significantly increased in the tumours after aCD47@CaCO $3 @$ fibrin treatment as compared to controls (Fig. 5b,c). Proliferation of $\mathrm{CD}^{+} \mathrm{T}$ cells was also increased, while the percentage of Tregs (CD $4^{+}$Foxp $3^{+} \mathrm{T}$ cells) was decreased (Fig. 5d and Supplementary Fig. 16). Immunofluorescence staining visually indicated the marked increase in macrophages and $\mathrm{CD} 8+\mathrm{T}$ cells in the residual tumours after aCD47@CaCO$@ @ f i b r i n$ therapy (Fig. 5e and Supplementary Fig. 17). Secretion of cytokines including IFN- $\gamma$, IL-6 and IL-12p70 further confirmed the effective innate and adaptive immune responses induced by aCD47@ $\mathrm{CaCO}_{3} @$ fibrin treatment (Fig. 5f).

\section{Immunotherapeutic gel for treating distant tumours}

With confirmation that aCD47@CaCO $3 @$ fibrin activates locally innate and adaptive immunity, we investigated whether local treatment with aCD47@CaCO $3 @ f i b r i n$ triggers systemic immune responses. B16F10 cancer cells were inoculated in the opposite flank of the primary tumour to mimic tumour metastasis. Primary tumours were then partially resected, and fibrin gel containing aCD47@ $\mathrm{CaCO}_{3}$ nanoparticles $\left(1 \mathrm{mg} \mathrm{CaCO}_{3}\right.$ per mouse; $50 \mu \mathrm{g}$ aCD47 per mouse) was sprayed into the tumour resection cavity
(Fig. 6a). Local tumour recurrence was inhibited by aCD47@ $\mathrm{CaCO}_{3} @$ fibrin treatment, and tumour growth at the opposite site was also reduced (Fig. $6 \mathrm{~b}-\mathrm{d}$ ). Consistent with these results, the numbers of M1-like TAMs and $\mathrm{CD}_{103}{ }^{+}$dendritic cells were significantly increased in tumours sprayed with aCD47@CaCO $\mathrm{CO}_{3} @$ fibrin (Fig. 6e,f), and $\mathrm{CD}^{+} \mathrm{T}$ cells were increased in both treated and distant tumours (Fig. $6 \mathrm{~g}$ and Supplementary Fig. 18). The increased $\mathrm{CD}^{+} \mathrm{T}$ cells in the distant tumour can be attributed to local crosspresentation of tumour antigens by macrophages and dendritic cells that trigger systemic antitumour immunity. Activation of the immune system was further confirmed by the peritumoral injection of aCD47@CaCO$@ @$ fibrin by a dual-syringe administration

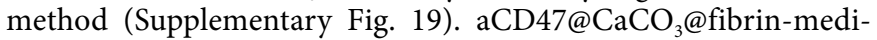
ated treatment effectively inhibited the growth of both local and distant tumours.

We further evaluated the combination efficacy of antiPD-1 (aPD1) and aCD47 co-delivered by the sprayed gel ${ }^{3,44-47}$. Similarly, the primary tumours were partially resected and the surgical sites were sprayed with different fibrin gels containing aCD47@ $\mathrm{CaCO}_{3}$ nanoparticles $(50 \mu \mathrm{g}$ aCD47 per mouse), aPD1@ $\mathrm{CaCO}_{3}$ nanoparticles (50 $\mu \mathrm{g}$ aPD1 per mouse) or aCD47\&aPD1@ $\mathrm{CaCO}_{3}$ nanoparticles $(25 \mu \mathrm{g}$ aCD47 per mouse; $25 \mu \mathrm{g}$ aPD1 per mouse). Encouragingly, a synergistic effect was achieved with aCD47\&aPD1@CaCO $@$ @fibrin treatment in inhibiting tumour recurrence after surgery and distant tumours, as shown in the bioluminescence imaging and growth profiles of local and distant tumours (Supplementary Fig. 20).

\section{Conclusion}

In summary, we have developed a simple post-surgical cancer immunotherapy strategy by spraying in situ formed therapeutic gel at the tumour resection site, which could facilitate reversal of the immunosuppressive TME and induce systemic immunological responses that inhibit both local recurrence and systemic development. $\mathrm{CaCO}_{3}$ nanoparticles embedded in the gel matrix could help release therapeutics in a controlled manner and modulate the acidic and inflamed tumour resection environment by scavenging $\mathrm{H}^{+}$, thereby promoting antitumour immune responses. Furthermore, the locally released aCD47 from $\mathrm{CaCO}_{3}$ nanoparticles blocked the 'don't eat me' signal associated with cancer cells, allowing cancer cell removal by macrophages. CD47 blockade also triggered the $\mathrm{T}$ cell mediated destruction of cancer cells due to the enhanced presentation of tumour-specific antigen by macrophages and dendritic cells. These results encourage the potential clinical translation of this method upon tumour resection. To this end, future evaluations in large animal models are expected to optimize drug dosage, particle amount and treatment frequency.

\section{Online content}

Any methods, additional references, Nature Research reporting summaries, source data, statements of data availability and associated accession codes are available at https://doi.org/10.1038/ s41565-018-0319-4.

Received: 11 May 2018; Accepted: 30 October 2018; Published online: 10 December 2018

\section{References}

1. Turajlic, S. \& Swanton, C. Metastasis as an evolutionary process. Science 352, 169-175 (2016).

2. Wang, C. et al. In situ activation of platelets with checkpoint inhibitors for post-surgical cancer immunotherapy. Nat. Biomed. Eng. 1, 0011 (2017).

3. Wang, C. et al. In situ formed reactive oxygen species-responsive scaffold with gemcitabine and checkpoint inhibitor for combination therapy. Sci. Transl. Med. 10, eaan3682 (2018). 
4. Tohme, S. et al. Neutrophil extracellular traps promote the development and progression of liver metastases after surgical stress. Cancer Res. 76, 1367-1380 (2016).

5. Baker, D., Masterson, T., Pace, R., Constable, W. \& Wanebo, H. The influence of the surgical wound on local tumor recurrence. Surgery 106 525-532 (1989).

6. Grivennikov, S. I., Greten, F. R. \& Karin, M. Immunity, inflammation and cancer. Cell. 140, 883-899 (2010).

7. Vakkila, J. \& Lotze, M. T. Inflammation and necrosis promote tumour growth. Nat. Rev. Immunol. 4, 641-648 (2004).

8. Albain, K. S. et al. Radiotherapy plus chemotherapy with or without surgical resection for stage III non-small-cell lung cancer: a phase III randomised controlled trial. The Lancet 374, 379-386 (2009).

9. Kwon, E. D. et al. Elimination of residual metastatic prostate cancer after surgery and adjunctive cytotoxic T lymphocyte-associated antigen 4 (CTLA-4) blockade immunotherapy. Proc. Natl Acad. Sci. USA 96 15074-15079 (1999)

10. Stephan, S. B. et al. Biopolymer implants enhance the efficacy of adoptive T-cell therapy. Nat. Biotechnol. 33, 97-101 (2015).

11. Pardoll, D. M. The blockade of immune checkpoints in cancer immunotherapy. Nat. Rev. Cancer 12, 252-264 (2012).

12. Wang, C., Ye, Y., Hu, Q., Bellotti, A. \& Gu, Z. Tailoring biomaterials for cancer immunotherapy: emerging trends and future outlook. Adv. Mater. 29, 1606036 (2017).

13. Chen, Q. et al. Photothermal therapy with immune-adjuvant nanoparticles together with checkpoint blockade for effective cancer immunotherapy. Nat. Commun. 7, 13193 (2016).

14. Gordon, S. Alternative activation of macrophages. Nat. Rev. Immunol. 3 23-35 (2003).

15. Calandra, T. \& Roger, T. Macrophage migration inhibitory factor: a regulator of innate immunity. Nat. Rev. Immunol. 3, 791-800 (2003).

16. Subramanian, S., Parthasarathy, R., Sen, S., Boder, E. T. \& Discher, D. E. Species- and cell type-specific interactions between CD47 and human SIRP $\alpha$. Blood 107, 2548-2556 (2006).

17. Edris, B. et al. Antibody therapy targeting the CD47 protein is effective in a model of aggressive metastatic leiomyosarcoma. Proc. Natl Acad. Sci. USA 109, 6656-6661 (2012)

18. Michaels, A. D. et al. CD47 blockade as an adjuvant immunotherapy for resectable pancreatic cancer. Clin. Cancer Res. 24, 1415-1425 (2018).

19. Ring, N. G. et al. Anti-SIRP $\alpha$ antibody immunotherapy enhances neutrophil and macrophage antitumor activity. Proc. Natl Acad. Sci. USA 114, 10578-10585 (2017)

20. Jaiswal, S. et al. CD47 is upregulated on circulating hematopoietic stem cells and leukemia cells to avoid phagocytosis. Cell 138, 271-285 (2009).

21. Kershaw, M. H. \& Smyth, M. J. Making macrophages eat cancer. Science 341, 41-42 (2013).

22. Sockolosky, J. T. et al. Durable antitumor responses to CD47 blockade require adaptive immune stimulation. Proc. Natl Acad. Sci. USA 113, 2646-2654 (2016).

23. Huang, Y., Ma, Y., Gao, P. \& Yao, Z. Targeting CD47: the achievements and concerns of current studies on cancer immunotherapy. J. Thorac. Dis. 9, E168-E174 (2017).

24. Herberman, R. R., Ortaldo, J. R. \& Bonnard, G. D. Augmentation by interferon of human natural and antibody-dependent cell-mediated cytotoxicity. Nature 277, 221-223 (1979).

25. Lin, E. Y. \& Pollard, J. W. Tumor-associated macrophages press the angiogenic switch in breast cancer. Cancer Res. 67, 5064-5066 (2007)

26. Mantovani, A., Sozzani, S., Locati, M., Allavena, P. \& Sica, A. Macrophage polarization: tumor-associated macrophages as a paradigm for polarized M2 mononuclear phagocytes. Trends Immunol. 23, 549-555 (2002).

27. Neubert, N. J. et al. T cell-induced CSF1 promotes melanoma resistance to PD1 blockade. Sci. Transl. Med. 10, eaan3311 (2018).

28. Lawrence, T. \& Natoli, G. Transcriptional regulation of macrophage polarization: enabling diversity with identity. Nat. Rev. Immunol. 11 750-761 (2011).

29. Wang, Y.-C. et al. Notch signaling determines the M1 versus M2 polarization of macrophages in antitumor immune responses. Cancer Res. 70, 4840-4849 (2010).

30. Chanmee, T., Ontong, P., Konno, K. \& Itano, N. Tumor-associated macrophages as major players in the tumor microenvironment. Cancers 6, $1670-1690$ (2014).
31. Baer, C. et al. Suppression of microRNA activity amplifies IFN- $\gamma$-induced macrophage activation and promotes anti-tumour immunity. Nat. Cell Biol. 18, 790-802 (2016).

32. Lewis, C. E., Harney, A. S. \& Pollard, J. W. The multifaceted role of perivascular macrophages in tumors. Cancer Cell. 30, 18-25 (2016).

33. Pyonteck, S. M. et al. CSF-1R inhibition alters macrophage polarization and blocks glioma progression. Nat. Med. 19, 1264-1272 (2013).

34. Martin, P. Wound healing-aiming for perfect skin regeneration. Science $\mathbf{2 7 6}$ 75-81 (1997).

35. Zhao, Y. et al. A preloaded amorphous calcium carbonate/doxorubicin@silica nanoreactor for ph-responsive delivery of an anticancer drug. Angew. Chem. Int. Ed. 54, 919-922 (2015).

36. Lu, Y., Aimetti, A. A., Langer, R. \& Gu, Z. Bioresponsive materials. Nat. Rev. Mater. 2, 16075 (2017)

37. Mi, P. et al. A pH-activatable nanoparticle with signal-amplification capabilities for non-invasive imaging of tumour malignancy. Nat. Nanotech. 11, 724-730 (2016).

38. Credo, R., Curtis, C. \& Lorand, L. $\mathrm{Ca}^{2+}$-related regulatory function of fibrinogen. Proc. Natl Acad. Sci. USA 75, 4234-4237 (1978).

39. Chen, Q. et al. A self-assembled albumin-based nanoprobe for in vivo ratiometric photoacoustic pH imaging. Adv. Mater. 27, 6820-6827 (2015).

40. Colegio, O. R. et al. Functional polarization of tumour-associated macrophages by tumour-derived lactic acid. Nature 513, 559-563 (2014).

41. Liu, Q. et al. Nanoparticle-mediated trapping of wnt family member 5A in tumor microenvironments enhances immunotherapy for B-Raf protooncogene mutant melanoma. ACS Nano 12, 1250-1261 (2018).

42. Rodell, C. B. et al. TLR7/8-agonist-loaded nanoparticles promote the polarization of tumour-associated macrophages to enhance cancer immunotherapy. Nat. Biomed. Eng. 2, 578 (2018).

43. Meyer, M. A. et al. Breast and pancreatic cancer interrupt IRF8-dependent dendritic cell development to overcome immune surveillance. Nat. Commun. 9, 1250 (2018)

44. Velu, V. et al. Enhancing SIV-specific immunity in vivo by PD-1 blockade. Nature 458, 206-210 (2009).

45. Tumeh, P. C. et al. PD-1 blockade induces responses by inhibiting adaptive immune resistance. Nature 515, 568-571 (2014).

46. Zou, W., Wolchok, J. D. \& Chen, L. PD-L1 (B7-H1) and PD-1 pathway blockade for cancer therapy: mechanisms, response biomarkers and combinations. Sci. Transl. Med. 8, 328rv324 (2016).

47. Ribas, A. \& Wolchok, J. D. Cancer immunotherapy using checkpoint blockade. Science 359, 1350-1355 (2018).

\section{Acknowledgements}

This work was supported by grants from start-up packages from UNC/NC state and UCLA, the Jonsson Comprehensive Cancer Center at UCLA, the Alfred P. Sloan Foundation (Sloan Research Fellowship), the National Key R\&D Program of China (2017YFA0205600), the Program for Guangdong Introducing Innovative and Enterpreneurial Teams (2017ZT07S054) and the National Natural Science Foundation of China (51728301). The authors thank L. Huang at UNC at Chapel Hill for providing the B16F10-Luc-GFP.

\section{Author contributions}

Q.C., G.D. and Z.G. conceived and designed the experiments. Q.C., C.W., X.Z., G.C., Q.H., Ji.W., D.W., Y.Z., H.L., Y.L., G.Y. and X.Z. performed the experiments and analysed data. Q.C., G.C., C.J., Ju.W., G.D. and Z.G. co-wrote the paper. All authors discussed the results and implications and edited the manuscript at all stages.

\section{Competing interests}

Z.G. and Q.C. have applied for patents related to this study. Z.G. is a scientific co-founder of ZenCapsule Inc.

\section{Additional information}

Supplementary information is available for this paper at https://doi.org/10.1038/ s41565-018-0319-4.

Reprints and permissions information is available at www.nature.com/reprints.

Correspondence and requests for materials should be addressed to Z.G.

Publisher's note: Springer Nature remains neutral with regard to jurisdictional claims in published maps and institutional affiliations.

(C) The Author(s), under exclusive licence to Springer Nature Limited 2018 


\section{Methods}

Materials, cell lines and animals. All chemicals were purchased from SigmaAldrich and used without any purification. Murine thrombin and fibrinogen were purchased from Molecular Innovations. aCD47 and aPD1 were purchased from Biolegend (cat. no. 127518, Clone, miap301; cat. no. 135233, Clone, 29F.1A12). The murine melanoma cell line B16F10 was purchased from UNC tissue culture facility. B16F10-luc cells were obtained from L. Huang at UNC. Cells were cultured in Dulbecco's modified Eagle medium (Gibco, Invitrogen) containing 10\% fetal bovine serum (Invitrogen) and $100 \mathrm{U} \mathrm{ml}^{-1}$ penicillin (Invitrogen) in an incubator at $37^{\circ} \mathrm{C}$ in $5 \% \mathrm{CO}_{2}$. Female $\mathrm{C} 57 \mathrm{BL} / 6$ mice (6-10 weeks) were purchased from the Jackson Lab. All mouse studies were carried out following protocols approved by the Institutional Animal Care and Use Committee at the University of North Carolina at Chapel Hill and North Carolina State University and complied with all relevant ethical regulations.

Preparation and characterization of aCD47@CaCO . $\mathrm{aCD} 47 @ \mathrm{CaCO}_{3}$ nanoparticles were prepared by chemical precipitation. Typically, $1 \mathrm{ml}$ of Tris- $\mathrm{HCl}$ buffer $(1 \mathrm{mM}, \mathrm{pH} 7.6)$ containing $100 \mathrm{mM} \mathrm{CaCl}_{2}$ was mixed with $1 \mathrm{ml} \mathrm{HEPES}$ saline buffer $(50 \mathrm{mM}, \mathrm{pH} 7.1, \mathrm{NaCl} 140 \mathrm{mM})$ containing $100 \mu \mathrm{g}$ aCD $47,10 \mathrm{mg}$ PEG-b-P(Glu) block copolymers (synthesized as previously described ${ }^{48}$ ) and $10 \mathrm{mM} \mathrm{Na}_{2} \mathrm{CO}_{3}$. The mixture was stirred for $12 \mathrm{~h}$ at $4{ }^{\circ} \mathrm{C}$. Excess ions, copolymers and antibodies were removed by centrifugation at 14,800 r.p.m. for $5 \mathrm{~min}$. The size distribution was measured by dynamic light scattering and morphology was evaluated by TEM (JEOL 2000FX). The elemental analysis of aCD47@CaCO (aCD47 was chelated gadolinium as previously described ${ }^{49}$ ) was characterized using analytical TEM (Titan). The amount of aCD47 encapsulated in $\mathrm{CaCO}_{3}$ was measured by enzyme-linked immunosorbent assay (ELISA) (rat IgG total ELISA kit, eBioscience, cat. no. 88-50490-22). The encapsulation efficiency (EE) and loading capacity (LC) of $\mathrm{CaCO}_{3}$ nanoparticles were calculated using the following formula: $\mathrm{EE}=(\mathrm{A}-\mathrm{B}) / \mathrm{A}$ and $\mathrm{LC}=(\mathrm{A}-\mathrm{B}) / \mathrm{C}$, where $\mathrm{A}$ is the feed amount of antibody, $\mathrm{B}$ is the free non-entrapped antibody and $\mathrm{C}$ is the total weight of particles.

Formation and characterization of aCD47@CaCO$@$ @fibrin. Fibrin gels were obtained by spraying equal volumes of fibrinogen $\left(10 \mathrm{mg} \mathrm{ml}^{-1}\right)$ containing aCD47@ $\mathrm{CaCO}_{3}$ and thrombin $\left(10 \mathrm{NIH} \mathrm{U} \mathrm{ml}^{-1}\right)$. For the control group without $\mathrm{CaCO}_{3}$ nanoparticles, $\mathrm{CaCl}_{2}$ was incorporated with the same amount of free $\mathrm{Ca}^{2+}$ compared with the solution with $\mathrm{CaCO}_{3}$ nanoparticles. The morphology of aCD47@CaCO $@$ @fibrin was characterized by cryo-SEM (JEOL 7600F, Gatan Alto). Encapsulation of aCD47@ $\mathrm{CaCO}_{3}$ in fibrin was further characterized by a confocal microscope (Zeiss LSM 710)

Release of aCD47 in vitro. The release of aCD47 was studied at $37^{\circ} \mathrm{C}$ in $\mathrm{PBS}$ at various $\mathrm{pH}$ values ( $\mathrm{pH} 6.5$ and 7.4). Released aCD47 was measured using a rat $\mathrm{IgG}$ total ELISA kit.

Release of aCD47 in vivo. To study the in vivo release of aCD47, Cy5.5-labelled free aCD47 or aCD47@ $\mathrm{CaCO}_{3}$ dispersed in PBS was injected at the tumour resection site. For gel administration, equal volumes of fibrinogen solution $\left(10 \mathrm{mg} \mathrm{ml}^{-1}\right)$ containing aCD47@ $\mathrm{CaCO}_{3}$ with the same dose of aCD47 (50 $\mu \mathrm{g}$ per mouse) and thrombin solution $\left(10 \mathrm{NIHU} \mathrm{ml}{ }^{-1}\right)$ were sprayed at the tumour resection site. Fluorescence imaging was monitored by an IVIS Spectrum imaging system (Perkin Elmer). To investigate whether the antibody was released from the nanoparticles or still encapsulated in the nanoparticles, we studied intratumoral antibody release behaviour using FITC-labelled $\mathrm{CaCO}_{3}$ nanoparticles. Tumours were collected at different time points after spraying the hydrogel, and the frozen tumour sections were stained with DAPI and rhodamine (TRITC)-conjugated donkey anti-rat IgG antibody.

In vitro phagocytosis assay. Bone-marrow-derived macrophage cells (BMDMs) were prepared as previously described ${ }^{50}$, stained with CellTracker Green (C7025, Thermo-Fisher Scientific), and cancer cells were stained with CellTracker DeepRed (C34565, Thermo-Fisher Scientific). Cancer cells $\left(4 \times 10^{5}\right)$ pre-blocked with IgG or aCD47 were co-cultured with macrophages $\left(1 \times 10^{5}\right)$ in serum-free medium. After incubation for $2 \mathrm{~h}$ at $37^{\circ} \mathrm{C}$, phagocytosis was studied by confocal microscopy (Zeiss LSM 710) and CytoFLEX flow cytometry (Beckman).

In vivo tumour models and treatment. To study the therapeutic effects of aCD47@ $\mathrm{CaCO}_{3} @$ fibrin, $1 \times 10^{6}$ fLuc-B16F10 cells were transplanted into the right flanks of mice. Ten days later, mice were randomly divided into five groups $(n=6-8)$ and tumours were resected, leaving $\sim 1 \%$ residual tumour to mimic residual micro tumours after surgery. Briefly, mice were anaesthetized in an induction chamber using isoflurane (up to $5 \%$ for induction; $1-3 \%$ for maintenance), and anaesthesia was maintained via a nose cone. Sterile instruments were used to remove $\sim 99 \%$ of the tumour. Immediately after surgery, fibrin gels with different formulations, including fibrin, IgG@CaCO $3 @ f i b r i n, a C D 47 @ f i b r i n$ and aCD47@CaCO $3 @ f i b r i n$ with calculated dosages of antibody, were sprayed on the surgical tumour bed by a dual-cartridge sprayer. The wound was then closed by an Autoclip wound clip system. For the distant tumour model, 7 days after $1 \times 10^{6} \mathrm{fLuc}-\mathrm{B} 16 \mathrm{~F} 10$ were transplanted into the right flank of mice, a second tumour as the distant tumour $\left(1 \times 10^{6} \mathrm{fLuc}-\mathrm{B} 16 \mathrm{~F} 10\right)$ was inoculated into the left flank of each mouse. Three days later, tumours in the right flank were partially resected and the immune therapeutic gel was sprayed on the surgical tumour bed. The tumour size was measured and calculated according to the following formula: width $^{2} \times$ length $\times 0.5$. The tumour was also observed using an in vivo bioluminescence imaging system. Ten minutes after intraperitoneal injection of D-luciferin (Thermo Scientific Pierce) in DPBS $\left(15 \mathrm{mg} \mathrm{ml}^{-1}\right)$ into each mouse at a dose of $10 \mu \mathrm{g} \mathrm{g}^{-1}$, mice were imaged using an IVIS Spectrum Imaging System (Perkin Elmer) for $5 \mathrm{~min}$. Regions of interest were quantified as the average radiance (photons $\mathrm{s}^{-1} \mathrm{~cm}^{-2} \mathrm{sr}^{-1}$ ) using Living Image software. Animals were euthanized when showing signs of imperfect health or when the size of their tumours exceeded $1.5 \mathrm{~cm}^{3}$.

Cytokine detection. The intratumour and plasma levels of IL-10 (Invitrogen, cat. no. BMS215-2), IL-12p70 (Invitrogen, cat. no. BMS6004TEN), IL-6 (Invitrogen, cat. no. BMS603-2), IFN- $\gamma$ (Invitrogen, cat. no. BMS606INST) and TNF- $\alpha$ (Invitrogen, cat. no. BMS607-3) were measured with ELISA kits according to the manufacturer's instructions. For detection of the local (tumour) concentration of cytokines, 5 days after spraying the fibrin gels, the tumour tissue was harvested and then homogenized in cold PBS buffer in the presence of digestive enzymes, forming single cell suspensions. For the plasma levels, serum samples were isolated from mice after different treatments and diluted for analysis.

Flow cytometry. Tumours collected from mice were divided into small pieces and homogenized in cold staining buffer to form single cell suspensions in the presence of digestive enzyme. Cells were stained with fluorescence-labelled antibodies CD45 (Biolegend, cat. no. 103108, clone 30-F11), CD11b (Biolegend, cat. no. 101208, clone M1/70), CD206 (Biolegend, cat. no. 141716, clone C068C2), F4/80 (Biolegend, cat. no. 123116, clone BM8), CD80 (Biolegend, cat. no. 104722, clone 16-10A1), Gr-1 (Biolegend, cat. no. 108412, clone RB6-8C5), CD3 (Biolegend, cat. no. 100204, clone 17A2), CD4 (Biolegend, cat. no. 100412, clone GK1.5), CD8 (Biolegend, cat. no. 140408, clone 53-5.8), Foxp3 (Biolegend, cat. no. 126404, clone MF-14), CD11c (Biolegend, cat. no. 117310, clone N418), CD86 (Biolegend, cat. no. 105008, clone GL-1), CD103 (Biolegend, cat. no. 121406, clone 2E7), Ly6C (Biolegend, cat. no. 128016, clone HK1.4) and Ly6G (Biolegend, cat. no. 127608 , clone 1A8) following the manufacturer's instructions. All antibodies were diluted 200 times. The stained cells were measured on a CytoFLEX flow cytometer (Beckman) and analysed by FlowJo software (version 10.0.7, TreeStar). The numbers presented in the flow cytometry analysis images are percentage based.

Immunofluorescence staining. Tumours were collected from the mice and snapfrozen in optimal cutting temperature medium. Tumour sections were cut using a cryotome, mounted on slides and stained with different primary antibodies: CD4 (Abcam, cat. no. ab183685), CD8 (Abcam, cat. no. ab22378) and F4/80 (Abcam, cat. no. ab100790) overnight at $4{ }^{\circ} \mathrm{C}$ following the manufacturer's instructions. Following the addition of fluorescently labelled secondary antibodies (goat anti-rat IgG (H + L; Thermo Fisher Scientific, cat. no. A18866) and goat anti-rabbit IgG $(\mathrm{H}+\mathrm{L}$; Thermo Fisher Scientific, cat. no. A32733)), the slides were analysed with a confocal microscope (Zeiss LSM 710). All antibodies used in the experiments were diluted 200 times.

Western blotting. Equal amounts of protein (measured using a bicinchoninic acid protein assay kit, BCA) were mixed with an equal volume of $2 \times$ Laemmli buffer and boiled at $95^{\circ} \mathrm{C}$ for $5 \mathrm{~min}$. After gel electrophoresis and protein transformation, anti-HIF1- $\alpha$ (28b) antibody at a dilution of 1:1,000 (Santa Cruz, cat. no. sc-13515) and anti- $\beta$-actin antibody (Abcam, cat. no. ab8226) at a 1:5,000 dilution were used as primary antibodies. The secondary antibody used for these blots was a goat antimouse antibody (Novus Biologicals, cat. no. NBP1-75151).

Statistical analysis. All results are presented as the mean \pm standard error of the mean (s.e.m.), as indicated. Tukey post-hoc tests and one-way ANOVA were used for multiple comparisons (when more than two groups were compared), and Student's $t$ test was used for two-group comparisons. Survival benefit was determined using a log rank test. All statistical analyses were carried out with Prism software package (PRISM 5.0; GraphPad Software, 2007). The threshold for statistical significance was $P<0.05$.

Reporting summary. Further information on research design is available in the Nature Research Reporting Summary linked to this article.

\section{Data availability}

The data that support the plots within this paper and other findings of this study are available from the corresponding author upon reasonable request.

\section{References}

48. Nishiyama, N. et al. Novel cisplatin-incorporated polymeric micelles can eradicate solid tumors in mice. Cancer Res. 63, 8977-8983 (2003).

49. Chen, Q. et al. An albumin-based theranostic nano-agent for dual-modal imaging guided photothermal therapy to inhibit lymphatic metastasis of cancer post surgery. Biomaterials 35, 9355-9362 (2014).

50. Lee, E. J. et al. Nanocage-therapeutics prevailing phagocytosis and immunogenic cell death awakens immunity against cancer. Adv. Mater. 30, 1705581 (2018). 